\title{
Negative operations on proofs and labels
}

\author{
Tatiana Yavorskaya (Sidon) *
}

June 6, 2005

\begin{abstract}
Logic of proofs $\mathcal{L P}$ introduced by $\mathrm{S}$. Artemov in 1995 describes properties of proof predicate " $t$ is a proof of $F$ " in the propositional language extended by atoms of the form $\llbracket t \rrbracket F$. Proof are represented by terms constructed by three elementary recursive operations on proofs.

In order to make the language more expressive we introduce the additional storage predicate with the intended interpretation " $x$ is a label for F". It can play the role of syntax encoding, so it is useful for specification of operations that require formula arguments.

In this paper we study operations on proofs and labels that can be specified in the extended language. First, we give a formal definition of an operation on proofs and labels. Then, for an arbitrary finite set of operations $\mathcal{F}$ the $\operatorname{logic} \mathcal{L} \mathcal{P}(\mathcal{F})$ is defined. We provide $\mathcal{L} \mathcal{P} \mathcal{S}(\mathcal{F})$ with symbolic semantics and arithmetical semantics.

The main result of the paper is the uniform completeness theorem for this family of logics with respect to the both types of semantics.
\end{abstract}

\section{Introduction}

In $[1,2]$, S. Artemov defined the Logic of Proofs $\mathcal{L P}$. It is formulated in the propositional language enriched by formulas of the form $\llbracket t \rrbracket F$ with the intended meaning " $t$ is a proof of $F$ ". Here $t$ is a proof term which represent arithmetical proofs. Proof terms are constructed from proof variables and

\footnotetext{
*The work is partially supported by grants from Russian Foundation for Basic Research, Utrecht University and Netherlands Organization for Scientific Research (NWO).
} 
proof constants by means of three functional symbols representing elementary computable operations on proofs. These operations are binary $\cdot$ and + and unary !. The Logic of Proofs $\mathcal{L P}$ is axiomatized over propositional calculus by the weak reflexivity principle and axioms for operations ".", "+" and "!"

$$
\begin{aligned}
& \llbracket t \rrbracket A \rightarrow A \\
& \llbracket t \rrbracket(A \rightarrow B) \rightarrow(\llbracket s \rrbracket A \rightarrow \llbracket t \cdot s \rrbracket B) \\
& \llbracket t \rrbracket A \rightarrow \llbracket t+s \rrbracket A, \quad \llbracket s \rrbracket A \rightarrow \llbracket t+s \rrbracket A \\
& \llbracket t \rrbracket A \rightarrow \llbracket ! t \rrbracket \llbracket t \rrbracket A
\end{aligned}
$$

weak reflexivity

application

nondeterministic choice

positive proof checker

and axiom necessitation rule, which allows to specify proof constants as proofs of the concrete axioms

$$
\overline{\llbracket a \rrbracket A}, \quad \text { where } a \text { is an axiom constant, } A \text { is an axiom of } \mathcal{L P} \text {. }
$$

The intended semantics for $\mathcal{L P}$ is formalized in Peano Arithnmetic PA; $\llbracket t \rrbracket F$ is interpreted by an arithmetical proof predicate which numerates theorems of PA. It is proved in [2] that $\mathcal{L P}$ is complete with respect to arithmetical interpretations based on multi-conclusion proof predicates (i.e. those where a proof can prove more than one proposition). It is also shown that the modal counterpart of $\mathcal{L P}$ is Gödel's provability logic $S 4$. This fact gives possibility to provide $S 4$ and, therefore, intuitionistic logic with the exact provability semantics.

In this paper we consider the language of $\mathcal{L P}$ as a convenient framework in which operations on proofs can be described and studied. To begin, let us look what operations on proofs can be represented by $\mathcal{L} \mathcal{P}$-terms. The answer is given in [1]. It is proved there that terms constructed by $\{\cdot,+, !\}$ together with proof constants suffices to represent all positive operations on arithmetical proofs which allow description in the language of $\mathcal{L} \mathcal{P}$. All these operations have proofs both as the arguments and the result, that is, they have the type

$$
\underbrace{\text { proof } \times \ldots \times \text { proof }}_{n} \rightarrow \text { proof. }
$$

Below are examples of other natural operations, which provide proofs, but require arguments of other types.

Negative proof checker. If we interpret $\square$ as provability in Peano Arithmetic then the following principle is valid $\neg \llbracket p \rrbracket A \rightarrow \square \neg \llbracket p \rrbracket A$. One can write down precisely the quantifier hidden in the provability operator $\square$ and obtain the following principle (with the parameters $p, A) \exists q(\neg \llbracket p \rrbracket A \rightarrow \llbracket q \rrbracket \neg \llbracket p \rrbracket A)$. 
The standard skolemization procedure gives the operation called negative proof checker which depends on two arguments: a proof $p$ and a formula $A$. This operation check $^{-}:$proof $\times$proposition $\rightarrow$ proof is specified as follows:

$$
\neg \llbracket p \rrbracket A \rightarrow \llbracket \operatorname{check}^{-}(p, A) \rrbracket \neg \llbracket p \rrbracket A .
$$

Axiom verifier. Let us choose an axiom of propositional logic, for example $A \rightarrow(B \rightarrow A)$. The formula $\square(A \rightarrow(B \rightarrow A))$ is valid under every arithmetical interpretation. The corresponding Skolem function depends on two formula arguments $A$ and $B$. That is, we can specify the operation $\mathrm{ax}_{1}$, called axiom verifier, of the type $\mathrm{ax}_{1}$ : proposition $\times$ proposition $\rightarrow$ proof by the principle

$$
\llbracket \operatorname{ax}_{1}(A, B) \rrbracket(A \rightarrow(B \rightarrow A)) .
$$

If we take a more nontrivial valid principle instead of the propositional axiom, then we obtain a more interesting operation which depends on formula arguments only. For example, the operation which finds the proof of weak reflexivity principle can be specified as follows

$$
\llbracket \operatorname{ax}_{r}(\llbracket p \rrbracket A) \rrbracket(\llbracket p \rrbracket A \rightarrow A) .
$$

The arithmetical proof of the weak reflexivity is not trivial, while in $\mathcal{L P}$ it is represented by a proof constant, which erases all information about this proof.

These examples provide motivation to develop the language, which is able to specify operations of the type

$$
\underbrace{\text { proof } \times \ldots \times \text { proof }}_{n} \times \underbrace{\text { proposition } \times \ldots \times \text { proposition }}_{k} \rightarrow \text { proof } .
$$

The approach taken in [9] is to extend propositional language by two predicates: the proof predicate of $\mathcal{L P}$ " $p$ is a proof of $F$ " and the additional storage predicate " $x$ is a label (or code) for $F$ " denoted by the formula $x \ni F$. So, we introduce a new sort of objects which is called labels. We assume that every label is assigned to a finite set of propositions. Now we can specify operations which depend on both proofs and labels. We are going to use labels everywhere when we need formula arguments. For example, the operations of negative proof checker and reflexivity checker can be specified as follows:

$$
\begin{aligned}
& x \ni A \wedge \neg \llbracket p \rrbracket A \rightarrow \llbracket \operatorname{check}^{-}(p, x) \rrbracket \neg \llbracket p \rrbracket A \\
& (x \ni \llbracket p \rrbracket A) \rightarrow \llbracket \operatorname{ax}_{r}(p, x) \rrbracket(\llbracket p \rrbracket A \rightarrow A) .
\end{aligned}
$$


Our approach is related to the one considered in $[3,4,5,6]$. In these papers single-conclusion proof predicates are studied, namely, those predicates for which every proof may prove at most one formula. In $[3,6]$ it is suggested to consider single-conclusion proofs as pointers (labels) for propositions: if $t$ is a (single-conclusion) proof of $A$, then one can use $t$ as a label for $A$. In [6] the language is enriched with special constructions which allow to carry out pattern matching.

This paper continues the research started in [9] in which positive operations on proofs and labels were considered, namely, those which use only positive information about proofs and labels.

The structure of our paper is the following. In section 2 we define what an operation on proofs and labels is. In 2.1 we define the language $\mathcal{L}_{0}$ in which operations on proofs will be specified and describe two types of semantics (symbolic and arithmetical) for $\mathcal{L}_{0}$. In 2.2 we briefly remind results from [9] concerning the minimal logic of proofs and labels $\mathcal{L} \mathcal{P} \mathcal{S}_{0}$. It describes the most general properties of storage and proof predicates and does not contain operations on proofs, however, $\mathcal{L} \mathcal{P} \mathcal{S}_{0}$ provides us with the framework for description of operations on proofs and labels. In 2.3 specifications of an operation on proofs are defined semantically. The syntactical criteria for a formula to specify an operation is proved; the set of specifications is shown to be decidable.

In section 3 we study logics which describe operations specified by schemas in the language of $\mathcal{L P} \mathcal{S}_{0}$. In 3.1 we define logics $\mathcal{L P} \mathcal{S}(\mathcal{F})$ where $\mathcal{F}$ stands for a finite collection of operations. We describe symbolic and arithmetical semantics for these logics and prove soundness with respect to both types of semantics. Section 3.2 is devoted to the proof of completeness of the logic $\mathcal{L P} \mathcal{S}(\mathcal{F})$ with respect to symbolic semantics. As a corollary of this theorem we obtain decidability and the complexity bounds, namely, satisfiability problem for all these logics is NP-complete. In 3.3 we prove arithmetical completeness of $\mathcal{L P S}(\mathcal{F})$. The both completeness theorems are uniform on the choice of $\mathcal{F}$. 


\section{Specification of operations on proofs and labels}

\subsection{Specification language $\mathcal{L}_{0}$ and its semantics}

Definition 2.1. The language $\mathcal{L}_{0}$ contains objects of three sorts: propositions, labels and proofs. It consists of variables of every sort:

propositional variables $S \operatorname{Var}=\left\{S_{1}, S_{2}, \ldots\right\}$

label variables $L$ Var $=\left\{x_{1}, x_{2}, \ldots\right\}$

proof variables $P$ Var $=\left\{p_{1}, p_{2}, \ldots\right\}$,

boolean connectives and two operational symbols

$\llbracket \cdot \rrbracket(\cdot)$ of the type proof $\rightarrow($ proposition $\rightarrow$ proposition $)$

$(\cdot) \ni(\cdot)$ of the type label $\rightarrow($ proposition $\rightarrow$ proposition $)$.

Formulas are constructed from sentence variables by means of boolean connectives and according to the rule: if $p$ is a proof variable, $x$ is a label variable and $F$ is a formula then $x \ni F$ and $\llbracket p \rrbracket F$ are formulas too. Formulas of the form $x \ni F$ and $\llbracket p \rrbracket F$ are called q-atomic.

For any formula $F$ by $S \operatorname{Var}(F), P \operatorname{Var}(F)$ and $L \operatorname{Var}(F)$ we denote the sets of all sentence variables, proof variables and label variables from $F$ respectively; $\operatorname{Var}(F)$ stands for $S \operatorname{Var}(F) \cup \operatorname{PVar}(F) \cup L \operatorname{Var}(F)$.

Informally speaking, the language $\mathcal{L}_{0}$ is supposed to describe the structure containing objects of three types: propositions (represented by formulas), labels (or names of propositions) (represented by label variables) and proofs (represented by proof variables). The structure is supplied with two predicates: a storage predicate between labels and propositions " $x$ is a label for a proposition $F "$ " (denoted by the formula $x \ni F$ ) and a proof predicate " $p$ is a proof of a proposition $F "$ (represented by the formula $\llbracket p \rrbracket F)$.

We assume that both the storage predicate $x \ni F$ and the proof predicate $\llbracket p \rrbracket F$ are recursive relations. We suppose that a finite set of propositions is assigned to every label, the function that recovers the corresponding set being given a label is total recursive, and there exists a total recursive function which for every proposition returns a label assigned to it. For every proof the set of propositions proved by it is finite and the function that maps proofs to the corresponding sets is total recursive. We are going to specify operations on proofs and labels in the language $\mathcal{L}_{0}$; in the informal semantics 
they correspond to total recursive functions which have labels and proofs as their arguments and return proofs.

Now let us give the formal definition of the symbolic semantics for the language $\mathcal{L}_{0}$.

Definition 2.2. A symbolic model $\mathcal{M}=(\#, v)$ consists of two objects: a binary relation $\# \subseteq(P \operatorname{Var} \cup L \operatorname{Var}) \times F m$ between label and proof variables and formulas and a truth evaluation of sentence variables $v: S V a r \mapsto$ \{true, false\}. Definition of the truth relation $\mathcal{M} \models A$ is inductive: for propositional variables $\mathcal{M} \models S$ iff $v(S)=$ true, $\models$ commutes with boolean connectives and

$$
\mathcal{M} \models(x \ni A) \rightleftharpoons \#(x, A) ; \quad \mathcal{M} \models \llbracket p \rrbracket A \rightleftharpoons \#(p, A) \text { and } \mathcal{M}=A .
$$

A model $\mathcal{M}=(\#, v)$ is called finite if the relation \# is finite and evaluation $v$ is primitive recursive.

Now we are going to formalize the intended semantics for $\mathcal{L}_{0}$ in Peano Arithmetic. We will interpret proof and storage operators by proof and storage predicates respectively.

Definition 2.3. A storage predicate $\operatorname{Str}(x, y)$ is an arithmetical $\Delta_{1}$ formula such that for every arithmetical sentence $\psi$ there exists $x$ such that $\operatorname{Str}(x, \psi)$, for every $n$ the set $\operatorname{Cont}(n) \rightleftharpoons\{m \mid \operatorname{Str}(n, m)\}$ is finite and the function $\lambda n .\ulcorner\operatorname{Cont}(n)\urcorner$ is total recursive.

A proof predicate $\operatorname{Prf}$ is an arithmetical $\Delta_{1}$ formula such that for every arithmetical formula $\varphi$ one has

$$
\mathbf{P A} \vdash \varphi \text { iff there exists } n \text { such that } \operatorname{Prf}(n,\ulcorner\varphi\urcorner) \text {, }
$$

A proof predicate $\operatorname{Prf}$ is single-conclusion if $\operatorname{Prf}\left(x, y_{1}\right) \wedge \operatorname{Prf}\left(x, y_{2}\right) \rightarrow y_{1}=y_{2}$ or multi-conclusion otherwise.

We say that $\operatorname{Prf}$ is normal if for every $n$ the set $\operatorname{Th} h_{\operatorname{Prf}}(n) \rightleftharpoons\{m \mid \operatorname{Prf}(n, m)\}$ is finite and the function $\lambda n . T h_{\operatorname{Prf}}(n)$ is total recursive. If $\operatorname{Prf}$ is multiconclusion then we also require that for every finite set of arithmetical theorems $\Gamma$ there exists a natural number $n$ such that $\Gamma \subseteq T h_{P r f}(n)$.

Example 2.4. The standard Gödel proof predicate and its natural multiconclusion version are arithmetical formulas proof $(x, y)$ and Proof $(x, y)$ which express the relations 
proof $(x, y) \rightleftharpoons " x$ is the Gödel number of a derivation in PA, $y$ is the Gödel number of the last formula in this derivation"

Proof $(x, y) \rightleftharpoons$ " $x$ is the Gödel number of a finite set of PA-derivations, $y$ is the Gödel number of the last formula in one of them".

The following two formulas are storage predicates; the second one is functional in $x$.

Store $(x, y) \rightleftharpoons$ " $x$ is the Gödel number of a finite set of formulas, $y$ is the Gödel number of one of them".

Store $_{1}(x, y) \rightleftharpoons$ "x is the Gödel number of a formula and $y=x "$.

Comment 2.5. Without loss of generality we may assume that from $\operatorname{Str}(x, y)$ it follows that $y$ is a Gödel number of an arithmetical sentence and the same holds for Prf. For every storage predicate $\operatorname{Str}$ the function $\iota x . \operatorname{Str}(x, y)$ which for every arithmetical sentence $\varphi$ returns $x$ such that $\operatorname{Str}(x,\ulcorner\varphi\urcorner)$ is total recursive. For every proof predicate $\operatorname{Prf}$ the similar function $\iota x . \operatorname{Prf}(x, y)$ is recursive but not total.

Definition 2.6. An arithmetical interpretation $*=\left(\operatorname{Str}, \operatorname{Prf},(\cdot)^{*}\right)$ for the language $\mathcal{L}_{0}$ has the following parameters:

- a storage predicate Str and a normal proof predicate Prf;

- an evaluation $(\cdot)^{*}$ that assigns natural numbers to label and proof variables and arithmetical sentences to propositional variables.

We extend evaluation $(\cdot)^{*}$ to all $\mathcal{L}_{0}$-formulas in the following way: it commutes with the boolean connectives and

$$
\begin{aligned}
& (\llbracket p \rrbracket A)^{*} \rightleftharpoons \exists x\left(1=1 \wedge x=\left\ulcorner A^{*}\right\urcorner \wedge \operatorname{Prf}\left(p^{*}, x\right)\right), \\
& (l \ni A)^{*} \rightleftharpoons \exists x\left(2=2 \wedge x=\left\ulcorner A^{*}\right\urcorner \wedge \operatorname{Str}\left(l^{*}, x\right)\right) .
\end{aligned}
$$

Comment 2.7. Note that PA $\vdash(\llbracket p \rrbracket A)^{*} \leftrightarrow \operatorname{Prf}\left(p^{*},\left\ulcorner A^{*}\right\urcorner\right)$ and, similarly, $\mathbf{P A} \vdash(l \ni A)^{*} \leftrightarrow \operatorname{Str}\left(l^{*},\left\ulcorner A^{*}\right\urcorner\right)$. We interpret the proof and storage predicates in the more sophisticated way because it makes the following problem decidable: being given $\operatorname{Prf}$, Str, an arithmetical formula $\varphi$ and an $\mathcal{L}_{0}$-formula $F$, decide whether there exists $(\cdot)^{*}$, such that $F^{*}=\varphi$. The values $v^{*}$ for $v \in \operatorname{Var}(F)$ are unique and can be found effectively. 


\subsection{The minimal logic of proofs and labels $\mathcal{L} \mathcal{P} \mathcal{S}_{0}$}

The logic $\mathcal{L} \mathcal{P} \mathcal{S}_{0}$ is very basic. However, when we turn to specification of operations in the language $\mathcal{L}_{0}$, the logic $\mathcal{L} \mathcal{P} \mathcal{S}_{0}$ helps to formulate a simple criterion for a formula to specify an operation and to provide all the parameters for it (see the next subsection for details). The logics which describe operations on proofs (see definition 3.1) are extensions of $\mathcal{L} \mathcal{P} \mathcal{S}_{0}$.

Definition 2.8. The minimal logic of proofs and labels $\mathcal{L P} \mathcal{S}_{0}$ is axiomatized by the following schemas ( $A$ is a formula, $p \in P$ Var ):

A0 classical propositional axioms

A1 $\llbracket p \rrbracket A \rightarrow A \quad$ weak reflexivity

The sole rule of inference is modus ponens.

The following results about $\mathcal{L} \mathcal{P} \mathcal{S}_{0}$ were proved in [9].

Theorem 2.9. $\mathcal{L} \mathcal{P} \mathcal{S}_{0}$ is sound and complete with respect to symbolic semantics, that is, for every formula $A$

1) if $\mathcal{L P S}_{0} \vdash A$ then for every model $\mathcal{M}$ one has $\mathcal{M} \models A$;

2) if $\mathcal{L} \mathcal{P} \mathcal{S}_{0} \forall A$ then there exists a finite model $\mathcal{M}$ such that $\mathcal{M} \forall A$.

Theorem 2.10. $\mathcal{L} \mathcal{P} \mathcal{S}_{0}$ is decidable. Satisfiability problem for $\mathcal{L} \mathcal{P} \mathcal{S}_{0}$ is NPcomplete.

Theorem 2.11. (Arithmetical soundness and completeness.)

For every $\mathcal{L} \mathcal{P} \mathcal{S}_{0}$ formula $A$ the following three propositions are equivalent:

1) $\mathcal{L} \mathcal{P} \mathcal{S}_{0} \vdash A$;

2) for every interpretation $*, \mathbf{P A} \vdash A^{*}$;

3) for every interpretation $*, A^{*}$ is true.

The completeness part of the last theorem asserts that if $\mathcal{L P}_{0} \forall A$ then there exists $*=\left(\operatorname{Prf}, \operatorname{Str},(\cdot)^{*}\right)$ such that $A^{*}$ is false. Below we prove the stronger version of the arithmetical completeness for $\mathcal{L P} \mathcal{S}_{0}$, namely, we show that Prf and Str can be taken independent on the choice of $A$.

Theorem 2.12. (Uniform arithmetical completeness.) There exist predicates $\operatorname{Prf}_{0}, \operatorname{Str}_{0}$ such that for every formula $A$ if $\mathcal{L} \mathcal{P} \mathcal{S}_{0} \forall A$ then $A^{*}$ is false for some $*=\left(\operatorname{Prf}_{0}, \operatorname{Str}_{0},(\cdot)^{*}\right)$. 
Proof. Let us define $\operatorname{Str}_{0}$ and $\operatorname{Prf} f_{0}$. We fix an injective Gödel numbering of the joint syntax of $\mathcal{L P} \mathcal{S}_{0}$ and PA. Consider all finite symbolic models for $\mathcal{L} \mathcal{P} \mathcal{S}_{0}$. Every such model $\mathcal{M}$ is defined by two finite objects: a relation \# and a truth evaluation $v$. Therefore, one can numerate finite models by natural numbers in such a way that the relation " $\mathcal{M}_{i} \models A$ " is primitive recursive in $i$ and $A$.

For every $i$ we define the evaluation $(\cdot)^{* i}$ as follows. Let $\xi_{i}$ be the $i$-th prime number. For every proof variable $p$, label variable $x$ and sentence variable $S_{j}$ we put

$$
p^{* i} \rightleftharpoons\left(\xi_{i}\right)^{\ulcorner p\urcorner}, \quad x^{* i} \rightleftharpoons\left(\xi_{i}\right)^{\ulcorner x\urcorner}, \quad S_{j}^{* i} \rightleftharpoons \begin{cases}j=j, & \text { if } v_{i}\left(S_{j}\right)=\text { true } \\ j=0, & \text { otherwise }\end{cases}
$$

if $\operatorname{Prf}$ and $S t r$ are given then for every formula $B$ and number $i$ the formula $B^{* i}$ is defined. Moreover, the function which produces $\left\ulcorner B^{* i}\right\urcorner$, being given $\operatorname{Prf}$, Str, $i$ and $\ulcorner B\urcorner$, is primitive recursive.

Now the appropriate storage and proof predicates can be defined simultaneously by the multiple fixed point equation. Let Proof and Store be predicates from the example 2.4; without loss of generality we assume that if $\operatorname{Store}(x, y) \vee \operatorname{Proof}(x, y)$ is true then $x$ is not a power of a prime number. The following formulas are provable in PA

$$
\begin{aligned}
\operatorname{Prf}_{0}(x, y) \leftrightarrow & \operatorname{Proof}(x, y) \vee \\
& \text { " } x=\left(\xi_{i}\right)^{\ulcorner p\urcorner} \text { and } y=\left\ulcorner B^{* i\urcorner} \text { and } \mathcal{M}_{i} \models \llbracket p \rrbracket B "\right. \\
\operatorname{Str}_{0}(x, y) \leftrightarrow & \operatorname{Store}(x, y) \vee \\
& \text { "x } x=\left(\xi_{i}\right)^{\ulcorner l\urcorner} \text { and } y=\left\ulcorner B^{* i\urcorner} \text { and } \mathcal{M}_{i} \models l \ni B "\right.
\end{aligned}
$$

Note that $\operatorname{Prf}_{0}, S t r_{0}$ are $\Delta_{1}$-formulas. It is easy to see that the interpretation $* i$ is injective, that is, for different formulas $B$ and $C$ we have $B^{i *} \neq C^{* i}$.

Let us prove that for every formula $C$ and number $i$

$$
\begin{aligned}
& \mathcal{M}_{i} \models C \Rightarrow \text { PA } \vdash C^{* i} ; \\
& \mathcal{M}_{i} \not \models C \Rightarrow \text { PA } \vdash \neg C^{* i} .
\end{aligned}
$$

Induction on the formula $C$. The case of propositional variables and boolean connectives is trivial. Suppose that $C$ has the form $\llbracket p \rrbracket B$. If $\mathcal{M}_{i} \models C$ then by the definition of $\operatorname{Prf}_{0}$ we conclude that PA $\vdash \operatorname{Prf} f_{0}\left(\left(\xi_{i}\right)^{\ulcorner p\urcorner},\left\ulcorner B^{* i}\right\urcorner\right)$ that is PA $\vdash C^{* i}$. If $\mathcal{M}_{i} \not \models C$ then PA $\vdash \neg \operatorname{Prf} f_{0}\left(\left(\xi_{i}\right)^{\ulcorner p\urcorner},\left\ulcorner B^{* i}\right\urcorner\right)$ because of the 
injectivity of the evaluation $(\cdot)^{* i}$. The remaining case when $C$ has the form $x \ni B$ can be treated similarly.

It is clear that $\operatorname{Str}_{0}$ is a storage predicate. Let us prove that $\operatorname{Prf}_{0}$ is a normal proof predicate. Since for every $i$ the model $\mathcal{M}_{i}$ is finite we conclude that for every $n$ the set $T h(n)$ is finite. The function $n \mapsto T h(n)$ is primitive recursive due to the choice of enumeration $\mathcal{M}_{i}$. The only thing we need to verify is that if $\operatorname{Prf}_{0}(n,\ulcorner\varphi\urcorner)$ is true then $\mathbf{P A} \vdash \varphi$. Suppose that $\operatorname{Prf}_{0}(n,\ulcorner\varphi\urcorner)$. By the definition of $\operatorname{Prf}_{0}$ there are two possibilities. The first case, when $\operatorname{Proof}(n,\ulcorner\varphi\urcorner)$ holds, is trivial. In the second case we have $n=\left(\xi_{i}\right)^{\ulcorner p\urcorner}$, $\varphi=B^{* i}$ and $\mathcal{M}_{i} \models \llbracket p \rrbracket B$. Then $\mathcal{M}_{i} \models B$ whence PA $\vdash B^{* i}$ by (1).

Finally, consider a formula $A$ such that $\mathcal{L P S}_{0} \forall A$. By theorem 2.9 there exists a finite symbolic model $\mathcal{M}_{i}=\left(\#_{i}, v_{i}\right)$ such that $\mathcal{M}_{i} \not \models A$. Take $*=\left(\operatorname{Prf}_{0}, \operatorname{Str}_{0},(\cdot)^{* i}\right.$; we conclude that $\mathbf{P A} \vdash \neg A^{*}$.

\subsection{Specification of operations in $\mathcal{L}_{0}$}

Our purpose is to formalize the general idea of what an operation on proofs and labels is. We start with the admissible inference rules, describes by the figures $A_{1}, \ldots, A_{n} \vdash F$ where all $A_{i}$ and $F$ are formulas of $\mathcal{L}_{0}$. This rule is admissible if for every arithmetical interpretation $*$ the formula $F^{*}$ is provable as soon as all $A_{i}^{*}$ are provable. We can describe this rule by a formula $\square A_{1} \wedge \ldots \wedge \square A_{n} \rightarrow \square F$ where $\square$ is interpreted as a provability operator $\exists x \operatorname{Prf}(x, \cdot)$. If we remove existential quantifiers from $\square$ 's in hypothesis then we obtain the formula $\llbracket p_{1} \rrbracket A_{1} \wedge \ldots \wedge \llbracket p_{n} \rrbracket A_{n} \rightarrow \square F$ which is true under every arithmetical interpretation. This formula specifies the operation which recovers a proof of $F$ being given proofs of all $A_{i}$ and possibly some additional information.

Our definition of the operation on proofs and labels extends the example considered above in three ways. Firstly, we add hypothesis of the form $x_{i} \ni C_{i}$ in order to describe more operations, for example, those which need formula arguments (see example 2.15). Secondly, we allow negative formulas of the form $\neg \llbracket q_{i} \rrbracket B_{i}$ and $\neg\left(y_{i} \ni D_{i}\right)$ in the hypothesis, so our operations may use negative information. Thirdly, we admit a kind of choice: if we can obtain $F$ using one of several sets of hypothesis $\delta_{1}, \ldots, \delta_{k}$, then we allow to describe an operation of the form $\delta_{1} \vee \ldots \vee \delta_{k} \rightarrow \square F$. Finally, we try to choose the definition invariant with respect to the particular choice of Prf, Str.

Roughly speaking, we call a specification of an operation on proofs and 
labels every arithmetical valid formula of the form $\delta \rightarrow \square F$ where $\delta$ is an arbitrary boolean combination of $q$-atomic formulas. However, in order to describe all parameters of the operation precisely, it is more convenient to have the formula $\delta$ in the disjunctive normal form with respect to its $q$-atomic subformulas. We will use special notation for the conjunction in the DNF for $\delta$ and for parts of these conjunctions, corresponding to positive and negative assumptions about proofs and labels. These notation are describes below.

Let $n$ be a natural number and $0 \leq i \leq n$. For every $i$ let us fix natural numbers $a_{i}, b_{i}, c_{i}$ and $d_{i}$. Then, we fix four lists of formulas in the language $\mathcal{L}_{0}$ of the lengthes $a_{i}, b_{i}, c_{i}$ and $d_{i}$ respectively:

$$
A_{i, 1}, \ldots, A_{i, a_{i}}, \quad B_{i, 1}, \ldots, B_{i, b_{i}}, \quad C_{i, 1}, \ldots, C_{i, c_{i}}, \quad D_{i, 1}, \ldots, D_{i, d_{i}} .
$$

two lists of proof variables of the lengthes $a_{i}$ and $b_{i}$

$$
p_{i, 1}, \ldots, p_{i, a_{i}}, \quad q_{i, 1}, \ldots, q_{i, b_{i}}
$$

and two lists of label variables of the lengthes $c_{i}$ and $d_{i}$

$$
x_{i, 1}, \ldots, x_{i, c_{i}}, \quad y_{i, 1}, \ldots, y_{i, d_{i}}
$$

We admit that some variables of the same type may coincide. By $\overrightarrow{p q}$ and $\overrightarrow{x y}$ we denote the lists of all variables $p_{i j}, q_{i j}\left(x_{i j}, y_{i j}\right.$ resp.) without repetitions. If $\Sigma$ is a set of formulas, then $\neg \Sigma$ stands for the set of negations of formulas from $\Sigma$. We define $\delta_{i} \rightleftharpoons \delta_{i}^{+} \cup \neg \delta_{i}^{-}$where

$$
\begin{array}{rlrl}
\delta_{i}^{+} & \rightleftharpoons \pi_{i}^{+} \cup \lambda_{i}^{+}, & \delta_{i}^{-} \rightleftharpoons \pi_{i}^{-} \cup \lambda_{i}^{-} \\
\pi_{i}^{+} \rightleftharpoons\left\{\llbracket p_{i j} \rrbracket A_{i j} \mid j \leq a_{i}\right\} & \pi_{i}^{-} \rightleftharpoons\left\{\llbracket q_{i j} \rrbracket B_{i j} \mid j \leq b_{i}\right\} \\
\lambda_{i}^{+} \rightleftharpoons\left\{x_{i j} \ni C_{i j} \mid j \leq c_{i}\right\} & \lambda_{i}^{-} \rightleftharpoons\left\{y_{i j} \ni D_{i j} \mid j \leq d_{i}\right\}
\end{array}
$$

Definition 2.13. Let $\delta_{i}$ be sets of formulas described above and let $F$ be an $\mathcal{L}_{0}$-formula. A specification of an operation on proofs and labels is a formula of the form

$$
\bigvee_{i=1}^{n}\left(\bigwedge \delta_{i}\right) \rightarrow \square F,
$$

which is true under every arithmetical interpretation where $\square F$ is interpreted as the provability formula $\exists x \operatorname{Prf}\left(x,\left\ulcorner F^{*}\right\urcorner\right)$.

Comment 2.14. By theorem 3.14 the formula (OP) is a specification iff $\mathcal{L P S}_{0} \vdash \bigvee_{i}\left(\bigwedge \delta_{i}\right) \rightarrow F$. Therefore, the set of specifications is decidable. 
Example 2.15. The basic operations of the logic of proofs $\mathcal{L P}$ correspond to the following specifications of operations in the sense of definition 2.13

$$
\begin{array}{ll}
\llbracket p \rrbracket\left(S_{1} \rightarrow S_{2}\right) \wedge \llbracket q \rrbracket S_{1} \rightarrow \square S_{2} & \text { application } \\
\llbracket p \rrbracket S \vee \llbracket q \rrbracket S \rightarrow \square S & \text { nondeterministic choice } \\
\llbracket p \rrbracket S \rightarrow \square \llbracket p \rrbracket S & \text { positive proof checker. }
\end{array}
$$

We can also specify other operations, for example

$$
\begin{array}{ll}
\left(x \ni S_{1}\right) \wedge\left(y \ni S_{2}\right) \rightarrow \square\left(S_{1} \rightarrow\left(S_{2} \rightarrow S_{1}\right)\right) & \text { axiom verifier. } \\
\neg \llbracket p \rrbracket S \rightarrow \square \neg \llbracket p \rrbracket S & \text { negative proof checker } \\
\llbracket p \rrbracket S_{1} \vee \llbracket q \rrbracket S_{2} \rightarrow \square\left(S_{1} \vee S_{2}\right) & \text { disjunction. }
\end{array}
$$

Comment 2.16. Definition 2.13 says that for every arithmetical interpretation $*$ formula $F^{*}$ is provable as soon as $\bigvee_{i}\left(\wedge \delta_{i}\right)^{*}$ holds. So, the formula (OP) specifies the algorithm which recovers the proof of $F^{*}$ under the assumption that one of $\left(\wedge \delta_{i}\right)^{*}$ is true. This algorithm works as follows. Given $\left(\operatorname{Prf}, \operatorname{Str},(\cdot)^{*}\right)$, reconstruct $F^{*}$ and $A^{*}$ for all $A \in \bigcup_{i} \delta_{i}$. Check whether $\left(\bigvee_{i}\left(\wedge \delta_{i}\right)\right)^{*}$ is true (this problem is decidable since $\left(\bigvee_{i}\left(\wedge \delta_{i}\right)\right)^{*}$ is a $\Delta_{1}$ formula). If no then return zero. If yes then consequently try $x=0,1,2, \ldots$ until $\operatorname{Prf}\left(x,\left\ulcorner F^{*}\right\urcorner\right)$ is true. Return the resulting $x$. The algorithm terminates since $\exists x \operatorname{Prf}\left(x,\left\ulcorner F^{*}\right\urcorner\right)$ is true.

Let us find out what are the parameters of the algorithm. In general, these are all the formulas from $\bigcup_{i} \delta_{i}$ and $F$. However, our purpose is to describe operations in such a way that the parameters of the corresponding algorithms are proofs and labels only, and they can be recovered in a uniform way from the syntax analysis of (OP) without taking the semantics into account. Namely, we would like to restrict the parameters by the list $\overrightarrow{p q}, \overrightarrow{x y}$. This idea is formalized in the following definition.

Definition 2.17. A specification (OP) is called complete if for every normal multi-conclusion predicate Prf and storage predicate Str there exists a total recursive function $f^{*}$ of arity $|\vec{p}|+|\overrightarrow{x y}|$ such that for every interpretation $\left(\operatorname{Prf}, \operatorname{Str},(\cdot)^{*}\right)$, the following formula is true

$$
\bigvee_{i}\left(\bigwedge \delta_{i}\right)^{*} \rightarrow \operatorname{Prf}\left(f^{*}\left(\overrightarrow{p q}^{*}, \overrightarrow{x y}^{*}\right),\left\ulcorner F^{*}\right\urcorner\right)
$$

In this case a specification axiom for the operation $f$ is the following formula in the extension of the language $\mathcal{L}_{0}$ by a new functional symbol $f$ :

$$
\bigvee_{i}\left(\bigwedge \delta_{i}\right) \rightarrow \llbracket f(\overrightarrow{p q}, \overrightarrow{x y}) \rrbracket F
$$


The arity of (OP) and $f$ is a pair of natural numbers $\left(n_{1}, n_{2}\right)$ where $n_{1}$ and $n_{2}$ are the numbers of variables in the lists $\overrightarrow{p q}$ and $\overrightarrow{x y}$ respectively. We say that the pair Prf, Str is compatible with Spec $(f)$ and function $f^{*}$ satisfies $\operatorname{Spec}(f)$ for Prf, Str if for every $(\cdot)^{*}$ formula (3) is true.

Comment 2.18. The definition of a complete specification says that we can specify precisely the parameters of the algorithm which reconstructs the proof of $F$. Now we are going to formulate and prove a syntactical criteria for the operation to be complete. Before we do that let us look at the examples of complete and non-complete specifications.

All operations of $\mathcal{L P}$ and axiom verifier from example 2.15 are complete. The corresponding specification axioms are the following formulas (we keep the notation of $\mathcal{L P}$ for the first three operations)

$$
\begin{aligned}
& \llbracket p \rrbracket\left(S_{1} \rightarrow S_{2}\right) \wedge \llbracket q \rrbracket S_{1} \rightarrow \llbracket p \cdot q \rrbracket S_{2} \\
& \llbracket p \rrbracket S \vee \llbracket q \rrbracket S \rightarrow \llbracket p+q \rrbracket S_{2} \\
& \llbracket p \rrbracket S \rightarrow \llbracket ! p \rrbracket \llbracket p \rrbracket S \\
& \left(x \ni S_{1}\right) \wedge\left(y \ni S_{2}\right) \rightarrow \llbracket \operatorname{ax}(x, y) \rrbracket\left(S_{1} \rightarrow\left(S_{2} \rightarrow S_{1}\right)\right) .
\end{aligned}
$$

Let us show that specification of negative proof checker is not complete. Suppose that $f^{*}$ is a total recursive function such that for every $(\cdot)^{*}$

$$
\neg \operatorname{Proof}\left(p^{*},\left\ulcorner S^{*}\right\urcorner\right) \rightarrow \operatorname{Proof}\left(f^{*}\left(p^{*}\right),\left\ulcorner\neg \operatorname{Proof}\left(p^{*},\left\ulcorner S^{*}\right\urcorner\right)\right\urcorner\right)
$$

Put $p^{*}=1$. Since for infinitely many formulas $\varphi$ one has $\neg \operatorname{Proof}(1,\ulcorner\varphi\urcorner)$, we conclude that $\operatorname{Th}\left(f^{*}(1)\right)$ is infinite, contradiction. Similar argument shows that specification of disjunction is incomplete too. However, we can turn these specifications into complete ones by adding new label arguments:

$$
\begin{aligned}
& \neg \llbracket p \rrbracket S \wedge x \ni S \rightarrow \llbracket \operatorname{check}^{-}(p, x) \rrbracket \neg \llbracket p \rrbracket S \\
& \left(\llbracket p \rrbracket S_{1} \vee \llbracket q \rrbracket S_{2}\right) \wedge x \ni S_{1} \wedge y \ni S_{2} \rightarrow \llbracket \operatorname{disj}(p, q, x, y) \rrbracket\left(S_{1} \vee S_{2}\right) .
\end{aligned}
$$

Lemma 2.19. Specification (OP) is complete iff for every $i=1, \ldots, n$ either $\mathcal{L P} \mathcal{S}_{0} \vdash \neg\left(\bigwedge \delta_{i}\right)$ or $\operatorname{Var}(F) \subseteq\{\overrightarrow{p q}, \overrightarrow{x y}\} \cup \operatorname{Var}\left(\delta_{i}^{+}\right)$.

Proof. $\quad(\Leftarrow) \quad$ Suppose that for every $i=1, \ldots, n$ either $\mathcal{L P} \mathcal{S}_{0} \vdash \neg\left(\bigwedge \delta_{i}\right)$ or $\operatorname{Var}(F) \subseteq\{\overrightarrow{p q}, \overrightarrow{x y}\} \cup \operatorname{Var}\left(\delta_{i}^{+}\right)$. We take any normal multi-conclusion proof predicate $\operatorname{Prf}$ and a storage predicate Str. Let us describe the algorithm which calculates the desired function $f^{*}$ for these predicates.

Suppose that the evaluation $\overrightarrow{p q x y}^{*}$ of variables $\overrightarrow{p q x y}$ is given. 
Step 1. Construct the set $T$ of arithmetical sentences which should be proven by $f^{*}\left(\overrightarrow{p q}^{*}, \overrightarrow{x y}^{*}\right)$ according to $\operatorname{Spec}(f)$. Initially $T=\emptyset$. For all $p_{i j}^{*}$ and $x_{i j}^{*}$ find $T h\left(p_{i j}^{*}\right)$ and $\operatorname{Cont}\left(x_{i j}^{*}\right)$ respectively. Take $i=1, \ldots, n$. For every $i$ do the following.

1. Find all partial arithmetical evaluations $(\cdot)^{\alpha}$ with the domain $\operatorname{Var}\left(\delta_{i}^{+}\right) \cup$ $\overrightarrow{p q x y}$ such that $(\overrightarrow{p q x y})^{\alpha}=(\overrightarrow{p q x y})^{*}$ and $A_{i j}^{\alpha} \in T h\left(p_{i j}^{*}\right)$ and $C_{i j}^{\alpha} \in$ $\operatorname{Cont}\left(x_{i j}^{*}\right)$ for all $j$ for the partial interpretation $\alpha=\left(\operatorname{Prf}, \operatorname{Str},(\cdot)^{\alpha}\right)$. The number of appropriate $(\cdot)^{\alpha}$ is finite and they can be found effectively, since all the sets $T h\left(p_{i j}^{*}\right)$ and $\operatorname{Cont}\left(x_{i j}^{*}\right)$ are finite.

2. Let $\delta_{i}^{ \pm}$be a maximal subset of $\delta_{i}^{-}$such that $\operatorname{Var}\left(\delta_{i}^{ \pm}\right) \subseteq \operatorname{Var}\left(\delta_{i}^{+}\right) \cup \overrightarrow{p q x y}$. For the every interpretation $\alpha$ from above check whether $\left(\bigwedge \neg \delta_{i}^{ \pm}\right)^{\alpha}$ is true. If no, then $\left(\bigwedge \delta_{i}\right)^{\alpha}$ is clearly false, do nothing. If yes, then add $F^{\alpha}$ into $T$. Go to the next $\alpha$ or the next $i$.

Comment. If $\left(\bigwedge \neg \delta_{i}^{ \pm}\right)^{\alpha}$ is true then the evaluation $(\cdot)^{\alpha}$ can be extended to an evaluation $(\cdot)^{\beta}$ of $\operatorname{Var}\left(\delta_{i}^{-}\right)$in such a way that $\left(\Lambda \neg \delta_{i}^{-}\right)^{\beta}$ is true. Indeed, every number proves or stores a finite set of formulas, therefore for every formula of the form $\llbracket q \rrbracket B$ where $B$ contains a variable $V \notin D o m(\alpha)$ there are infinitely many ways to extend $\alpha$ to $V$ so that $\llbracket q \rrbracket B$ is false in the resulting interpretation. The same for formulas of the form $y \ni D$. Therefore, in this case $\mathcal{L} \mathcal{P} \mathcal{S}_{0} \forall \neg\left(\bigwedge \delta_{i}\right)$ whence by the condition of the lemma $\operatorname{Var}(F) \subseteq$ $\operatorname{Var}\left(\delta_{i}^{+}\right) \cup \overrightarrow{p q x y}$. Thus, $\operatorname{Var}(F) \subseteq \operatorname{Dom}(\alpha)$ and $F^{\alpha}$ coincides with $F^{\beta}$. Also note that $\left(\delta_{i}\right)^{\beta}$ is true, whence $F^{\alpha}$ is provable according to the definition of OP.

Step 2. After we looked through all $i$, the resulting set $T$ is finite and consists of provable sentences. The algorithm returns $\mu n . T \subseteq T h(n)$. It terminates since $\operatorname{Prf}$ is a normal multi-conclusion proof predicate.

$(\Rightarrow) \quad$ Prove by contradiction. Suppose that there exists $i_{0}$ such that $\mathcal{L} \mathcal{P} \mathcal{S}_{0} \forall \neg\left(\bigwedge \delta_{i_{0}}\right)$ and $\operatorname{Var}(F) \nsubseteq \overrightarrow{p q x y} \cup \operatorname{Var}\left(\delta_{i_{0}}^{+}\right)$. By the uniform completeness theorem for $\mathcal{L} \mathcal{P} \mathcal{S}_{0}$ there exists an interpretation $*=\left(\operatorname{Prf}_{0}, \operatorname{Str}_{0},(\cdot)^{*}\right.$, where $\operatorname{Prf}_{0}$ is a normal multi-conclusion proof predicate and $\left(\bigwedge \delta_{i_{0}}\right)^{*}$ is true. Let $V$ be a variable from $\operatorname{Var}(F)$ such that $V \notin \overrightarrow{p q x y} \cup \operatorname{Var}\left(\delta_{i_{0}}^{+}\right)$. Consider all evaluations $(\cdot)^{\alpha}$ which coincide with $(\cdot)^{*}$ on all variables except $V$. Note that $(\cdot)^{\alpha}$ and $(\cdot)^{*}$ coincide on $\overrightarrow{p q x y} \cup \operatorname{Var}\left(\delta_{i_{0}}^{+}\right)$whence $\left(\bigwedge \delta_{i_{0}}^{+}\right)^{\alpha}$ is true. Since every number can prove or store only finitely many formulas, there are infinitely many evaluations $\alpha$ for which the formula $\left(\bigwedge \neg \delta_{i_{0}}^{-}\right)^{\alpha}$ is true and, therefore, 
$\delta_{i_{0}}^{\alpha}$ is true. According to the specification axiom, $f^{*}\left(\overrightarrow{p q}^{*}, \overrightarrow{x y}^{*}\right)$ should prove formulas $F^{\alpha}$ for all those $\alpha$. So, $f^{*}\left(\overrightarrow{p q}^{*}, \overrightarrow{x y}^{*}\right)$ proves infinitely many formulas, contradiction.

In the rest of the paper we consider only complete specifications. Note that if $\mathcal{L} \mathcal{P} \mathcal{S}_{0} \vdash \neg\left(\bigwedge \delta_{i}\right)$ then one can delete $\delta_{i}$ from the OP, the resulting formula is again a complete specification. In order to simplify the exposition in what follows we suppose that $\mathcal{L} \mathcal{P} \mathcal{S}_{0} \nvdash \neg\left(\bigwedge \delta_{i}\right)$ for every $i$. The following definition describes classes of specifications we are going to deal with.

Definition 2.20. A specification (OP) describes a positive operation if for every $i$ the set $\delta_{i}^{-}$is empty. A specification is called q-stable if for every $i$ all $q$-atomic subformulas of $F$ either belong to $\delta_{i}^{-}$or occur as subformulas in $\delta_{i}^{+}$, that is $q \operatorname{SubFm}(F) \subseteq\left(\delta_{i}^{-} \cup q \operatorname{SubFm}\left(\delta_{i}^{+}\right)\right)$. A specification is called descriptive if for every $i$ from $(y \ni D) \in \lambda_{i}^{-}$it follows that there is exists a renaming of label variables $\theta$ such that $(y \ni D) \theta \in \operatorname{SubFm}\left(\lambda_{i}^{+}\right)$and from $\llbracket q \rrbracket B \in \pi_{i}^{-}$it follows that $\operatorname{Var}(B) \cup q \operatorname{SubFm}(B) \subseteq \operatorname{SubFm}\left(\lambda_{i}^{+}\right)$.

Example 2.21. The following specifications are complete but not $q$-stable:

$$
\begin{aligned}
& \left(x \ni \llbracket p \rrbracket S_{1}\right) \wedge \llbracket q \rrbracket S_{2} \rightarrow \square\left(\llbracket p \rrbracket S_{2} \rightarrow \llbracket q \rrbracket S_{2}\right) \\
& \llbracket p \rrbracket S \rightarrow \square(\llbracket p \rrbracket(S \rightarrow S) \rightarrow \llbracket p \rrbracket S) \\
& (x \ni \llbracket p \rrbracket S) \rightarrow \square(\llbracket p \rrbracket(S \wedge \neg S) \rightarrow \llbracket p \rrbracket S) \\
& \left(x \ni \llbracket p \rrbracket S_{1}\right) \wedge\left(y \ni S_{2}\right) \rightarrow \square\left(\llbracket p \rrbracket S_{2} \rightarrow S_{2}\right)
\end{aligned}
$$

In the first two examples we can replace $q$-atoms in the conclusion which do not occur in premises by new propositional variables and add a label for this variable to the premise in order to make the new specification complete. As the result, we obtain a $q$-stable specification, and our original specification is a substitutional instance of the new one. However, we cannot do this in the last example.

The following specifications are $q$-stable, but not descriptive, the first two do not satisfy the conditions for variables occurring in $\pi_{i}^{-}$; for the last one the condition for $\lambda_{i}^{-}$does not hold:

$$
\begin{aligned}
& \llbracket p \rrbracket S \wedge \neg \llbracket q \rrbracket S \rightarrow \square \neg \llbracket q \rrbracket S \\
& \llbracket p \rrbracket(S \rightarrow S) \wedge \neg \llbracket q \rrbracket S \rightarrow \square \neg \llbracket q \rrbracket S \\
& \neg(y \ni(x \ni A)) \wedge(x \ni A) \rightarrow \square \neg(y \ni(x \ni A)) .
\end{aligned}
$$




\section{Logic of operations on proofs and labels}

\section{1 $\quad$ Logic $\mathcal{L P S}(\mathcal{F})$ and its semantics}

Let $(\mathrm{OP})_{i}(i=1, \ldots, n)$ be complete specifications of the types $\left(a_{i}, b_{i}\right)$. Let $\mathcal{F}=f_{1}, \ldots, f_{n}$ be the list of distinct functional symbols of the corresponding types proof ${ }^{a_{i}} \times$ label $^{b_{i}} \rightarrow$ proof. By $\mathcal{L}(\mathcal{F})$ we denote the extension of the language $\mathcal{L}_{0}$ by these functional symbols. Terms of the language $\mathcal{L}(\mathcal{F})$ are generated from proof and label variables by functional symbols: all proof variables are terms and if $f$ is a functional symbol of the type $(a, b), t_{1}, \ldots, t_{a}$ are proof terms and $x_{1}, \ldots, x_{b}$ are label variables then $f\left(t_{1}, \ldots, t_{a}, x_{1}, \ldots, x_{b}\right)$ is a proof term. Formulas are defined similarly with formulas of $\mathcal{L} \mathcal{P} \mathcal{S}_{0}$ with the only difference in the case of the proof operator: if $t$ is a term and $F$ is a formula then $\llbracket t \rrbracket F$ is a formula. We denote the sets of terms and formulas by $\operatorname{Tm}(\mathcal{F})$ and $F m(\mathcal{F})$ respectively.

A substitution for this language is a mapping which assigns proof terms to proof variables, label variables to label variables and formulas to sentence variables. We assume that substitutions have finite domains.

Definition 3.1. The logic of operations $f_{1}, \ldots, f_{n}$ denoted by $\mathcal{L P} \mathcal{S}(\mathcal{F})$ is the extension of the minimal logic $\mathcal{L} \mathcal{P} \mathcal{S}_{0}$ by formulas $\operatorname{Spec}\left(f_{i}\right)$ as new schemas of axioms: if $\sigma$ is a substitution, then $(\operatorname{Spec}(f)) \sigma$ is an axiom of $\mathcal{L P} \mathcal{S}(\mathcal{F})$.

In [9] we considered the logic of positive $q$-stable specifications. We proved that in this case $\mathcal{L P S}(\mathcal{F})$ is arithmetically complete. Also, the logics of proof positive specifications was provided with the appropriate symbolic semantics and the corresponding completeness theorem was proved. In this paper we prove similar results for the logic of all descriptive $q$-stable specifications including negative ones.

Let us describe symbolic semantics for $\mathcal{L P} \mathcal{S}(\mathcal{F})$. The definition below is an adjustment of symbolic semantics for $\mathcal{L P S}_{0}$ (see definition 2.2) to meet specifications from $\mathcal{F}$.

Definition 3.2. A symbolic model $\mathcal{M}=(\#, v)$ for $\mathcal{L P S}(\mathcal{F})$ consists of two objects: a binary relation \# between $\operatorname{LVar} \cup \operatorname{Tm}(\mathcal{F})$ and formulas which is called a proof assignment and a truth evaluation of sentence variables $v$. The relation \# should be compatible with operations $\mathcal{F}$, namely, for every 
operation $f \in \mathcal{F}$ and formula $G$ the following holds:

if there exists a substitution $\sigma$ such that $G=F \sigma$ and

$$
\begin{gathered}
\exists i \forall j\left(\#\left(p_{i j} \sigma, A_{i j} \sigma\right), \#\left(x_{i j} \sigma, C_{i j} \sigma\right), \neg \#\left(q_{i j} \sigma, B_{i j} \sigma\right), \neg \#\left(y_{i j} \sigma, D_{i j} \sigma\right)\right) \\
\text { then } \#(f(\overrightarrow{p q} \sigma, \overrightarrow{x y} \sigma), G) .
\end{gathered}
$$

We define the truth relation $\mathcal{M} \models A$ by induction on $A$ : for propositional variables $\mathcal{M} \models S$ iff $v(S)=$ true, $\models$ commutes with boolean connectives and

$$
\mathcal{M} \models x \ni A \rightleftharpoons \#(x, A), \quad \mathcal{M} \models \llbracket t \rrbracket A \rightleftharpoons \#(t, A) \text { and } \mathcal{M} \models A .
$$

For every $e \in L \operatorname{Var} \cup \operatorname{Tm}(\mathcal{F})$ we denote $\#(e)=\{F \in F m(\mathcal{F}) \mid \#(e, F)\}$. A model $\mathcal{M}=(\#, v)$ is called finitely generated if

- the set $\{x \in P \operatorname{Var} \cup L \operatorname{Var} \mid \#(x) \neq \emptyset\}$ is finite; for all $e \in L \operatorname{Var} \cup \operatorname{Tm}(\mathcal{F})$ the sets \# $(e)$ are finite;

- the reverse of (4) does not hold for a finite number of terms;

- $v$ is a primitive recursive function.

Comment 3.3. Suppose that $f \in \mathcal{F}, \sigma$ is a substitution and $\mathcal{A}_{i j}, \mathcal{B}_{i j}, \mathcal{C}_{i j}$ and $\mathcal{D}_{i j}$ are sets of $\mathcal{L}(\mathcal{F})$-formulas. By $f_{\sigma}\left(\mathcal{A}_{i j}, \mathcal{B}_{i j}, \mathcal{C}_{i j}, \mathcal{D}_{i j}\right)$ we denote the set

$$
\left\{F \sigma \mid \exists i \forall j\left(A_{i j} \sigma \in \mathcal{A}_{i j}, B_{i j} \sigma \notin \mathcal{B}_{i j}, C_{i j} \sigma \in \mathcal{C}_{i j}, D_{i j} \sigma \notin \mathcal{D}_{i j}\right)\right\} .
$$

Condition (4) can be formulated as follows: for every term $f(\overrightarrow{t s}, \overrightarrow{z w})$

$$
\bigcup_{\sigma}\left\{f_{\sigma}(\#(\vec{p} \sigma), \#(\vec{q} \sigma), \#(\vec{x} \sigma), \#(\vec{y} \sigma)) \mid(\overrightarrow{p q x y}) \sigma=\overrightarrow{t s z w}\right\} \subseteq \#(t)
$$

For a finitely generated model there exists a finite set of terms for which the inclusion above cannot be replaced by equality. Informally speaking, a model $\mathcal{M}=(\#, v)$ is finitely generated if the relation \# is completely defined after it is defined for a finite set of terms, and these terms correspond to finite sets of formulas. Note that if $\mathcal{M}$ is finitely generated then the relation $\mathcal{M} \models B$ and the function $I_{\mathcal{M}}(t) \rightleftharpoons\{B \mid \mathcal{M} \models \llbracket t \rrbracket B\}$ are primitive recursive.

It is proves in [9] that if $\mathcal{F}$ consists of positive $q$-stable operations then the $\operatorname{logic} \mathcal{L} \mathcal{P} \mathcal{S}(\mathcal{F})$ is sound and complete with respect to symbolic models described above. Namely, for every formula $A$ one has $\mathcal{L P} \mathcal{S}(\mathcal{F}) \vdash A$ iff $\mathcal{M} \models$ $A$ for every finitely generated model $\mathcal{M}$. The semantical counterpart for the logic of descriptive $q$-stable operations is provided by so-called reflexive models. 
Definition 3.4. A model $\mathcal{M}=(\#, v)$ is reflexive if for every term $t$ and formula $A$ from $\#(t, A)$ it follows that $\mathcal{M}=A$.

Theorem 3.5. (Soundness.) Suppose that $\mathcal{F}$ consists of complete specifications. If $\mathcal{L P} \mathcal{S}(\mathcal{F}) \vdash A$ then for every reflexive model $\mathcal{M}$ it is true that $\mathcal{M} \models A$.

Proof. Standard induction on the derivation of $A$ in $\mathcal{L P} \mathcal{S}(\mathcal{F})$. The only nontrivial thing here is to verify that all substitutional examples of the specification axioms are true. Consider a specification axiom $\bigvee_{i}\left(\bigwedge \delta_{i}\right) \rightarrow$ $\llbracket f(\overrightarrow{p q}, \overrightarrow{x y}) \rrbracket F$. Suppose that for a substitution $\sigma$ and a number $i$ one has that $\mathcal{M} \models\left(\bigwedge \delta_{i}\right) \sigma$. By the definition of the truth relation in a symbolic model the following conditions hold for all $j$ : \# $\left(p_{i j} \sigma, A_{i j} \sigma\right)$, \# $\left(x_{i j} \sigma, C_{i j} \sigma\right)$, $\neg \#\left(y_{i j} \sigma, D_{i j} \sigma\right)$, and either $\neg \#\left(q_{i j} \sigma, B_{i j} \sigma\right)$ or $\mathcal{M} \not \forall B_{i j} \sigma$. In the later case since $\mathcal{M}$ is reflexive we have $\neg \#\left(q_{i j} \sigma, B_{i j} \sigma\right)$.

Since \# is compatible with $f$, we obtain \# $(f(\overrightarrow{p q} \sigma, \overrightarrow{x y} \sigma), F)$. Moreover, note that every $\mathcal{L} \mathcal{P} \mathcal{S}(\mathcal{F})$-model is an $\mathcal{L} \mathcal{P} \mathcal{S}_{0}$-model (if we treat proof terms as atomic objects like proof variables). By the definition of an operation on proofs we have that $\mathcal{L} \mathcal{P} \mathcal{S}_{0} \vdash \bigwedge \delta_{i} \rightarrow F$, whence from $\mathcal{M} \models \bigwedge \delta_{i} \sigma$ it follows that $\mathcal{M} \models F \sigma$. Therefore, $\mathcal{M}=\llbracket f(\overrightarrow{p q} \sigma, \overrightarrow{x y} \sigma) \rrbracket F \sigma$.

Let us describe arithmetical semantics of $\mathcal{L P S}(\mathcal{F})$.

Definition 3.6. Consider the language $\mathcal{L}(\mathcal{F})$ where $\mathcal{F}=f_{1}, \ldots, f_{n}$ is a finite list of operations on proofs specified by the corresponding axioms $\operatorname{Spec}\left(f_{i}\right)$. An arithmetical interpretation $*=\left(\operatorname{Str}, \operatorname{Prf}, \mathcal{F}^{*},(\cdot)^{*}\right)$ of this language has the following parameters:

- a pair $(S t r, \operatorname{Prf})$ of a storage and a proof predicate compatible with all operations $f_{1}, \ldots, f_{n}$;

- $\mathcal{F}^{*}=\left\{f_{1}^{*}, \ldots, f_{n}^{*}\right\}$ where $f_{i}^{*}$ are total recursive functions satisfying $\operatorname{Spec}\left(f_{i}\right)$;

- an evaluation $(\cdot)^{*}$ that assigns natural numbers to label and proof variables and arithmetical sentences to propositional variables.

Arithmetical interpretation can be extended to all terms and formulas of the language $\mathcal{L} \mathcal{P} \mathcal{S}(\mathcal{F})$ in the usual way. For terms we put $f(\vec{t}, \vec{x})^{*} \rightleftharpoons f^{*}\left(\vec{t}^{*}, \vec{p}^{*}\right)$. For formulas we define $F^{*}$ similarly with 2.6 
Logic $\mathcal{L P S}(\mathcal{F})$ is sound and complete with respect to the described semantics. We formulate and prove soundness here and leave completeness until 3.3

Theorem 3.7. (Arithmetical soundness.) $\quad$ For every formula $A$ if $\mathcal{L P S}(\mathcal{F}) \vdash$ $A$ then for every interpretation $*$ of the language $\mathcal{L}(\mathcal{F})$ one has $\mathbf{P A} \vdash F^{*}$.

Proof. Induction on the derivation of $A$ in $\mathcal{L P S}(\mathcal{F})$.

\subsection{Completeness with respect to symbolic models}

Theorem 3.8. Completeness. Suppose that $\mathcal{F}$ consists of descriptive $q$ stable specifications. If $\mathcal{L P S}(\mathcal{F}) \forall A$ then there exists a finitely generated reflexive model $\mathcal{M}$ such that $\mathcal{M} \forall \neq A$.

We suppose that $\mathcal{L P S}(\mathcal{F}) \forall A$ and construct a reflexive finitely generated model $\mathcal{M}$ such that $\mathcal{M} \forall A$. Proof consists of three steps. On Step 1 we construct the set of formulas $\operatorname{Adeq}(A)$ whose truth values can affect the truth value of our formula $A$. This is done by the saturation algorithm. On step 2 we define a so-called partial model for formulas from $\operatorname{Adeq}(A)$ in which $A$ does not hold. We find this model via reduction of $\operatorname{Adeq}(A)$ to pure propositional language. On step 3 we extend the partial model from step 2 to the model which refutes $A$. We show that the resulting model is finitely generated and reflexive. Without loss of generality we assume that variables from $\operatorname{Var}(A)$ do not occur in specification schemes.

Step 1: Saturation algorithm. Being given $A$ it constructs a set of formulas $\operatorname{Adeq}(A)$ which is called an adequate set. We need the following notation:

$$
\operatorname{AdeqTm}(A):=\{t \theta \mid t \in \operatorname{SubTm}(A), \theta: \operatorname{LVar}(A) \rightarrow \operatorname{LVar}(A)\} .
$$

Everywhere in this step $\sigma$ ranges over substitutions with the domain $\operatorname{Dom}(\sigma) \subseteq$ $\operatorname{Var}\left(\delta_{i}^{+}\right)$and values for proof and label variables from $\operatorname{AdeqTm}(A)$ and $\operatorname{LVar}(A)$ respectively. The saturation algorithm works as follows:

1. Initialization. Calculate $\operatorname{AdeqTm}(A)$ and put

$$
\operatorname{Adeq}_{-1}(A):=\{B \theta, \neg B \theta \mid B \in \operatorname{SubFm}(A), \theta: \operatorname{LVar}(A) \rightarrow \operatorname{LVar}(A)\}
$$


2. Construct the set $A d e q_{0}(A)$ as follows. Initially $A d e q_{0}(A)=A d e q_{-1}(A)$. Look through all specification axioms $\operatorname{Spec}(f) \in \mathcal{F}$; every such axiom has the form $\bigvee_{i}\left(\bigwedge \delta_{i}\right) \rightarrow \llbracket f(\vec{p}, \vec{x}) \rrbracket F$. For every $i$ find all substitutions $\sigma$ for which $\lambda_{i}^{+} \sigma \subseteq \operatorname{Adeq}_{-1}(A)$. Extend $\operatorname{Adeq}_{0}(A)$ by $\operatorname{SubFm}\left(\delta_{i}^{-} \sigma\right)$.

3. Let $t_{1}, \ldots, t_{N}$ be the list of all terms from $\operatorname{AdeqTm}(A)$ with the property: if $t_{i} \in \operatorname{SubTm}\left(t_{j}\right)$ then $i<j$. For every $l=1, \ldots, N$ calculate the set of formulas $\operatorname{Adeq}_{l}(A)$ as follows.

(a) Initially $\operatorname{Adeq}_{l}(A):=\operatorname{Adeq}_{l-1}(A)$.

(b) Take the term $t_{l}$, if it is a proof variable then do nothing. If it has the form $f(\overrightarrow{t s}, \overrightarrow{z w})$ then take the specification axiom $\operatorname{Spec}(f)$; it has the form $\bigvee_{i}\left(\bigwedge \delta_{i}\right) \rightarrow \llbracket f(\overrightarrow{p q}, \overrightarrow{x y}) \rrbracket F$. For every $i$ find all substitutions $\sigma$ such that $(\overrightarrow{p q x y}) \sigma=\overrightarrow{t s z w}$ and $\delta_{i}^{+} \sigma \subseteq \operatorname{Adeq}_{l-1}(A)$. Extend $\operatorname{Adeq}_{l}(A)$ by $\operatorname{SubFm}\left(\llbracket t_{l} \rrbracket(F \sigma)\right)$.

4. Put $\operatorname{Adeq}(A):=\operatorname{Adeq}_{N}(A)$.

Lemma 3.9. 1. $\operatorname{Adeq}_{0}(A) \backslash A d e q_{-1}(A)$ consists of formulas of the form $\llbracket t \rrbracket E$ or formulas with the boolean connective as a principle symbol.

2. For every $l=0, \ldots, N$ the following assertions are true

(a) $\operatorname{Adeq}_{l}(A)$ is closed under subformulas.

(b) For every substitution $\sigma$ if $\lambda_{i}^{+} \sigma \subseteq A d e q_{l}$ then $\delta_{i}^{-} \sigma \subseteq A d e q_{l}$.

(c) If $G \in \operatorname{Adeq}_{l+1}(A) \backslash \operatorname{Adeq}_{l}(A)$ then $G$ either has the form $\llbracket t_{l+1} \rrbracket E$ or $G$ is a boolean combination of $q$-atomic formulas and sentence variables from $\operatorname{Adeq}_{l}(A)$.

Proof. Let us prove item 1. Suppose that $G \in A d e q_{0}(A) \backslash A d e q_{-1}(A)$. Then there exists a specification axiom and a substitution $\sigma$ such that $\delta_{i}^{+} \sigma \subseteq$ $\operatorname{Adeq}_{-1}(A)$ and $G \in \operatorname{SubFm}\left(\delta_{i}^{-} \sigma\right)$, that is, either $G \in \operatorname{SubFm}\left(\lambda_{i}^{-} \sigma\right)$ or $G \in \operatorname{SubFm}\left(\pi_{i}^{-} \sigma\right)$. By the definition of a descriptive operation, in the first case there exists a formula $(x \ni C) \in \lambda_{i}^{+}$and a renaming of label variables $\theta: L \operatorname{Var}(A) \mapsto \operatorname{LVar}(A)$ such that $G \in \operatorname{SubFm}((x \ni C) \theta \sigma)$. Therefore, $G \in \operatorname{SubFm}\left(\lambda_{i}^{+} \theta \sigma\right)$ whence $G \in \operatorname{Adeq}_{-1}(A)$. In the second case $G \in \operatorname{SubFm}((\llbracket q \rrbracket B) \sigma)$ where $\llbracket q \rrbracket B \in \pi_{i}^{-}$. There are three possibilities:

- $G=(\llbracket q \rrbracket B) \sigma=\llbracket q \sigma \rrbracket(B \sigma)$; item 1 trivially holds. 
- $G=E \sigma$, where $E \in \operatorname{SubFm}(B \sigma)$. The case of a boolean $E$ is trivial. If $E$ is a sentence variable or $q$-atomic formula, then according to the definition of a $q$-stable descriptive operation we conclude that $E \in$ $\operatorname{SubFm}\left(\delta_{i}^{+}\right)$whence $E \sigma \in \operatorname{Adeq}_{-1}(A)$.

- $G \in \operatorname{SubFm}\left(S_{i} \sigma\right)$, where $S_{i} \in S \operatorname{Var}(B)$. By the definition of a descriptive operation $S_{i} \in S \operatorname{Var}\left(\delta_{i}^{+}\right)$; therefore $\operatorname{SubFm}\left(S_{i} \sigma\right) \subseteq \operatorname{Adeq}_{-1}(A)$, thus $G \in \operatorname{Adeq}_{-1}(A)$.

Now we will prove propositions of item 2 by joint induction on $l$.

Induction base. By definition, $\operatorname{Adeq}_{0}(A)$ is closed under subformulas, so $2(\mathrm{a})$ is obvious. For 2(b) suppose that for some specification axiom, number $i$ and substitution $\sigma$ one has $\delta_{i}^{+} \sigma \subseteq \operatorname{Adeq}_{0}(A)$. By item $1, \lambda_{i}^{+} \sigma \subseteq \operatorname{Adeq}_{-1}(A)$. Since $\operatorname{Var}\left(\delta_{i}^{-}\right) \backslash\{\vec{q}, \vec{y}\} \subseteq \operatorname{Var}\left(\lambda_{i}^{+}\right)$, this substitution $\sigma$ was considered on step 2 of the saturation algorithm and $\operatorname{SubFm}\left(\delta_{i}^{-} \sigma\right)$ was added to $\operatorname{Adeq}_{0}(A)$.

For item 2(c) suppose that $G \in \operatorname{Adeq}_{1}(A) \backslash \operatorname{Adeq}_{0}(A)$. Then there exist a specification axiom $\operatorname{Spec}(f)$, a number $i$ and a substitution $\sigma$ such that $\delta_{i}^{+} \sigma \subseteq \operatorname{Adeq}_{0}(A), f(\overrightarrow{p q} \sigma, \overrightarrow{x y} \sigma)=t_{1}$ and $G \in S u b F m\left(\llbracket t_{1} \rrbracket(F \sigma)\right)$. By item 2(b) we obtain $\delta_{i}^{-} \sigma \subseteq \operatorname{Adeq}_{0}(A)$. There are three possible cases for $G$.

- $G=\llbracket t_{1} \rrbracket(F \sigma)$; this case is trivial.

- $G=E \sigma$, where $E \in \operatorname{SubFm}(F \sigma)$. The case of a boolean $E$ is trivial. If $E$ is a sentence variable or a $q$-atomic formula, then according to the definition of a $q$-stable descriptive operation we conclude that $E \in$ $\operatorname{SubFm}\left(\delta_{i}^{+}\right) \cup \delta_{i}^{-}$whence $E \sigma \in \operatorname{Adeq}_{0}(A)$.

- $G \in \operatorname{SubFm}\left(S_{i} \sigma\right)$ where $S_{i} \in S \operatorname{Var}(F)$. Since operation $f$ is complete, by lemma $2.19 S_{i} \in S \operatorname{Var}\left(\delta_{i}^{+}\right)$. Therefore $\operatorname{SubFm}\left(S_{i} \sigma\right) \subseteq \operatorname{Adeq}_{0}(A)$.

Induction step. For $2\left(\right.$ a) note that $\operatorname{Adeq}_{l+1}(A)$ is closed under subformulas, since both $\operatorname{Adeq}_{l}(A)$ and the set of additional formulas are, the first one by the induction hypothesis and the second one by the construction.

For 2(b) suppose that for some $i$ and $\sigma$ one has $\lambda_{i}^{+} \sigma \subseteq \operatorname{Adeq}_{l+1}(A)$. Then by the induction hypothesis for 2(c) this yields $\lambda_{i}^{+} \sigma \subseteq \operatorname{Adeq}_{l}(A)$ whence by the induction hypothesis for $2(\mathrm{~b}) \lambda_{i}^{-} \sigma \subseteq \operatorname{Adeq}_{l}(A)$.

Induction step for $2(\mathrm{c})$ is similar to the induction base. End of proof.

Lemma 3.10. Suppose that $t \in \operatorname{AdeqTm}(A), t=f(\overrightarrow{t s}, \overrightarrow{z w})$, and $\sigma$ is a substitution such that $(\overrightarrow{p q x y}) \sigma=\overrightarrow{t s z w}$. Suppose that there exists $i$ such that $\left(\delta_{i}\right) \sigma \subseteq \operatorname{Adeq}(A)$. Then $\llbracket t \rrbracket(F \sigma) \in \operatorname{Adeq}(A)$. 
Proof. Since $f(\overrightarrow{t s}, \overrightarrow{z w}) \in \operatorname{AdeqTm}(A)$ we have that $f(\overrightarrow{t s}, \overrightarrow{z w})$ coincides with some of the terms $t_{l}$. For all $j$ terms $t_{i j}$ are subterms of $t_{l}$. Thus, they occur in the list of terms of $\operatorname{AdeqTm}(A)$ with the numbers smaller than $l$ and they are processed by the saturation algorithm earlier. Then, by item $2(\mathrm{c})$ of lemma 3.9, all formulas $\llbracket t_{i j} \rrbracket\left(A_{i j} \sigma\right)$ belong to $\operatorname{Adeq}_{l-1}(A)$. From the same lemma we obtain that all formulas $\left(z_{i j} \ni C_{i j} \sigma\right)$ belong to $\operatorname{Adeq}_{0}(A)$, therefore, $\delta_{i}^{+} \sigma \subseteq \operatorname{Adeq}_{l-1}(A)$. From item 2(b) of lemma 3.9 we conclude that $\delta_{i}^{-} \sigma \subseteq \operatorname{Adeq}_{l-1}(A)$; therefore after step $l$ of the saturation algorithm is performed $\llbracket f(\overrightarrow{t s}, \overrightarrow{z w}) \rrbracket(F \sigma) \in \operatorname{Adeq}_{l}(A)$.

\section{Step 2. Partial model.}

Definition 3.11. Suppose that $L V \subseteq L V a r, S V \subseteq S$ Var and $T$ is a set of terms such that $L \operatorname{Var}(T) \subseteq L V$ and $T$ is close under subterms and renaming of label variables in $L V$. By $F m(S V, L V, T)$ we denote the set of all formulas $F$ for which $L \operatorname{Var}(F) \subseteq L V, S \operatorname{Var}(F) \subseteq S V$ and $\operatorname{SubTm}(F) \subseteq T$.

A partial model $\mathcal{M}_{p}=\left(\#_{p}, v_{p}\right)$ for $F m(S V, L V, T)$ consists of two objects: $\#_{p}$ is a relation between $T \cup L V$ and $F m(S V, L V, T) ; v_{p}$ is a truth evaluation of propositional variables from $S V$. The relation $\#_{p}$ should satisfy condition (4) restricted to terms from $T$, namely, for every operation $f \in \mathcal{F}$, substitution $\sigma$ and number $i$ if $f(\overrightarrow{p q} \sigma, \overrightarrow{x y} \sigma) \in T$ and $\#_{p}\left(p_{i j} \sigma, A_{i j} \sigma\right), \#_{p}\left(x_{i j} \sigma, C_{i j} \sigma\right)$, $\neg \# p_{p}\left(q_{i j} \sigma, B_{i j} \sigma\right), \neg \#_{p}\left(y_{i j} \sigma, D_{i j} \sigma\right)$ for all $j$ then \# $(f(\overrightarrow{p q} \sigma, \overrightarrow{x y} \sigma), F \sigma)$.

The relation " $\mathcal{M}_{p} \models F$ " is defined for $F \in F m(S V, L V, T)$ similarly with ordinary models. The definition of a finitely generated partial model and a reflexive partial model is the restriction of similar definitions for ordinary models to the sets $S V, L V, T$ and $F m(S V, L V, T)$.

For any formula $A$ we denote $F m(S \operatorname{Var}(A), \operatorname{LVar}(A), \operatorname{AdeqTm}(A))$ by $F m_{A}$.

Lemma 3.12. If $\mathcal{L} \mathcal{P} \mathcal{S}(\mathcal{F}) \forall A$ then there exists a finitely generated reflexive partial model $\mathcal{M}_{p}$ for $F m_{A}$ such that $\mathcal{M}_{p} \not \models A$.

Proof. Suppose that $\mathcal{L P S}(\mathcal{F}) \not \forall A$. Run the saturation algorithm on the input $A$; the result is the set $\operatorname{Adeq}(A) \subseteq F m_{A}$. For every term $t \in \operatorname{AdeqTm}(A)$ let us reserve a fresh proof variable $p_{t}$. Suppose that $F \in F m_{A}$. By $F^{0}$ we denote the result of replacing all uppermost occurrences of terms in $F$ by the corresponding proof variables, namely,

$$
(\llbracket t \rrbracket F)^{0} \rightleftharpoons \llbracket p_{t} \rrbracket F^{0} .
$$


Let $A X(A)$ stand for the set of specification axioms whose all $q$-atomic subformulas belong to $\operatorname{Adeq}(A)$ (here we replace an axiom $\bigvee_{i}\left(\bigwedge \delta_{i}\right) \rightarrow \llbracket f(\overrightarrow{p q}, \overrightarrow{x y}) \rrbracket F$ by a finite set of axioms $\left(\bigwedge \delta_{i}\right) \rightarrow \llbracket f(\overrightarrow{p q}, \overrightarrow{x y}) \rrbracket F$ for all $i$. For every $F \in F m_{A}$ define

$$
\widetilde{F} \rightleftharpoons((\bigwedge A X(A)) \rightarrow F)^{0}
$$

Since $\mathcal{L} \mathcal{P} \mathcal{S}(\mathcal{F}) \forall A$ we conclude that $\mathcal{L P S}_{0} \forall \widetilde{A}$ (otherwise the reverse substitution of terms $t$ for the corresponding proof variables $p_{t}$ transforms the derivation of $\widetilde{A}$ in $\mathcal{L} \mathcal{P} \mathcal{S}_{0}$ into the derivation of $A$ in $\mathcal{L P S}(\mathcal{F})$. According to theorem 2.9 there exists a model $\mathcal{M}_{0}=\left(\#_{0}, v_{0}\right)$ for $\mathcal{L} \mathcal{P} \mathcal{S}_{0}$ such that $\mathcal{M}_{0} \not \neq \widetilde{A}$.

Now we defined the desired partial model $\mathcal{M}_{p}$ as follows: for every $x \in$ $L \operatorname{Var}(A), S \in S \operatorname{Var}(A)$ and $t \in \operatorname{AdeqTm}(A)$ put

$$
\#_{p}(x, B) \text { iff } \#_{0}\left(x, B^{0}\right), \quad \#_{p}(t, B) \text { iff } \mathcal{M}_{0}=\llbracket p_{t} \rrbracket B^{0}, \quad v_{p}(S) \rightleftharpoons v_{0}(S) .
$$

By induction on the formula $F \in F m_{A}$ one can show that $\mathcal{M}_{p} \models F \Longleftrightarrow$ $\mathcal{M}_{0} \models F^{0}$. Since $\mathcal{M}_{0} \not \models \widetilde{A}$ we conclude that $\mathcal{M}_{p} \not \models A$. Let us prove now, that $\mathcal{M}_{0}$ is a reflexive finitely generated partial model.

Suppose that $t \in \operatorname{AdeqTm}(A)$ where $t=f(\overrightarrow{t s}, \overrightarrow{z w})$ and $\sigma$ is a substitution such that $(\overrightarrow{p q x y}) \sigma=\overrightarrow{t s z w}$. Suppose that there exists $i$ such that for all $j$ one has

$$
\#_{p}\left(t_{i j}, A_{i j} \sigma\right), \quad \neg \#_{p}\left(s_{i j}, B_{i j} \sigma\right), \quad \#_{p}\left(z_{i j}, C_{i j} \sigma\right), \quad \neg \#_{p}\left(w_{i j}, D_{i j} \sigma\right) .
$$

By definition of $\#_{p}$ it follows that

$$
\mathcal{M}_{0} \models \llbracket p_{t_{i j}} \rrbracket\left(A_{i j} \sigma\right)^{0}, \quad\left(z_{i j} \ni C_{i j} \sigma\right)^{0}, \quad \mathcal{M}_{0} \forall \models \llbracket p_{s_{i j}} \rrbracket\left(B_{i j} \sigma\right)^{0}, \quad\left(w_{i j} \ni D_{i j} \sigma\right)^{0} .
$$

From the definition of $\mathcal{M}_{0}$ it immediately follows that $\llbracket t_{i j} \rrbracket\left(A_{i j} \sigma\right) \in \operatorname{Adeq}(A)$ and $\left(z_{i j} \ni C_{i j} \sigma\right) \in \operatorname{Adeq}(A)$ for all $j$. Then by lemmas 3.10 and 3.9 we conclude that $\llbracket f(\overrightarrow{t s}, \overrightarrow{z w}) \rrbracket(F \sigma) \in \operatorname{Adeq}(A)$ and $\delta_{i}^{-} \sigma \subseteq \operatorname{Adeq}(A)$. Therefore, $\left(\bigwedge \delta_{i} \rightarrow \llbracket t \rrbracket F\right) \sigma$ belongs to $A X(A)$ whence the formula $\left(\left(\bigwedge \delta_{i} \sigma\right) \rightarrow \llbracket t \rrbracket(F \sigma)\right)^{0}$ is true in $\mathcal{M}_{0}$. As we mentioned above, $\mathcal{M}_{0} \models\left(\wedge \delta_{i} \sigma\right)^{0}$. Therefore, $\mathcal{M}_{0} \models$ $\llbracket p_{t} \rrbracket(F \sigma)^{0}$ whence by the definition of $\#_{p}$ we conclude that $\#_{p}(t, F \sigma)$. So, $\mathcal{M}_{p}$ is a partial model.

It is clear that $\mathcal{M}_{p}$ is finitely generated since the set $\operatorname{AdeqTm}(A)$ is finite and for every term $t \in \operatorname{AdeqTm}(A)$ the set $\#_{p}(t)$ is finite. Let us prove that $\mathcal{M}_{p}$ is reflexive. Suppose that $\#_{p}(t, F)$. By the definition of $\#_{p}$ this 
yields $\mathcal{M}_{0} \models \llbracket p_{t} \rrbracket F^{0}$ whence by the definition of a model for $\mathcal{L} \mathcal{P} \mathcal{S}_{0}$ one gets $\mathcal{M}_{0} \models F^{0}$. Therefore, $\mathcal{M}_{p} \models F$.

\section{Step 3. Completion.}

Lemma 3.13. (Completion.) Let $\mathcal{M}_{p}=\left(\#_{p}, v_{p}\right)$ be a partial model for $F m(S V, L V, T)$. Then there exists a model $\mathcal{M}=(\#, v)$ such that for every formula $F \in F m(S V, L V, T)$ one has $\mathcal{M} \models F$ iff $\mathcal{M}_{p}=F$. Moreover, if $\mathcal{M}_{p}$ is finitely generated, then $\mathcal{M}$ is, and if $\mathcal{M}_{p}$ is reflexive then $\mathcal{M}$ is.

Proof. We define \# as follows: for all $x \in L \operatorname{Var} \cup P \operatorname{Var}$ and for all $t \in T$

$$
\#(x, F) \text { iff } \#_{p}(x, F), \quad \#(t, F) \text { iff } \#_{p}(t, F) .
$$

For the remaining terms define \# by induction on the complexity of terms:

$$
\#(f(\overrightarrow{t s}, \overrightarrow{z w}))=\bigcup_{\sigma}\left\{f_{\sigma}(\#(\overrightarrow{p q} \sigma), \#(\overrightarrow{x y} \sigma)) \mid(\overrightarrow{p q x y}) \sigma=\overrightarrow{t s z w}\right\}
$$

Then let us define $v$ : for every sentence variable $S$ put

$$
v(S)=\text { true iff } v_{p}(S)=\text { true. }
$$

By induction on the formula $F \in F m(S V, L V, T)$ one can easily check that $\mathcal{M} \models F$ iff $\mathcal{M}_{p} \models F$.

Let us show that the resulting $\mathcal{M}$ is a model. Namely, we take any term $f(\overrightarrow{t s}, \overrightarrow{z w})$ and a substitution $\sigma$ such that $(\overrightarrow{p q x y}) \sigma=\overrightarrow{t s z w}$. We have to check that as soon as for all $j$ one has $\#\left(t_{i j}, A_{i j} \sigma\right), \neg \#\left(s_{i j}, B_{i j} \sigma\right), \#\left(z_{i j}, C_{i j} \sigma\right)$ and $\neg \#\left(w_{i j}, D_{i j} \sigma\right)$ then $\#(f(\overrightarrow{t s}, \overrightarrow{z w}), F \sigma)$. The case when $f(\overrightarrow{t s}, \overrightarrow{z w}) \notin T$ is trivial by the definition of \#. Suppose that $f(\overrightarrow{t s}, \overrightarrow{z w}) \in T$. Then for all $j$ we have $A_{i j} \sigma, C_{i j} \sigma \subseteq F m(S V, L V, T)$. Since $T$ is closed under subterms and $L \operatorname{Var}(T) \subseteq L V$, we get $\overrightarrow{t s} \subseteq T$ and $\overrightarrow{z w} \subseteq L V$. The operation $f$ is descriptive, therefore $\operatorname{Var}\left(\left\{B_{i j}, D_{i j} \mid j\right\}\right) \subseteq \operatorname{Var}\left(\lambda_{i}^{+}\right)$whence $B_{i j} \sigma, D_{i j} \sigma \in F m(S V, L V, T)$ for all $j$. Since $\mathcal{M}_{p}$ is a partial model for $\operatorname{Fm}(S V, L V, T)$ we conclude that $\#_{p}(f(\overrightarrow{t s}, \overrightarrow{z w}), F \sigma)$ whence $\#(f(\overrightarrow{t s}, \overrightarrow{z w}), F \sigma)$.

Let us show that $\mathcal{M}$ is finitely generated as soon as $\mathcal{M}_{p}$ is. The only thing which we have to verify here is the following: for every term $t$ the set $\#(t)$ is finite. Induction on $t$. The case when $t \in T$ or $t \in P \operatorname{Var}$ is trivial. Suppose that $t=f(\overrightarrow{t s}, \overrightarrow{z w})$ and $t \notin T$. Then according to the definition of \# we have that

$$
\#(t)=\bigcup_{\sigma}\left\{f_{\sigma}(\#(\overrightarrow{t s}), \#(\overrightarrow{z w}) \mid(\overrightarrow{p q x y}) \sigma=\overrightarrow{t s z w}\}\right.
$$


that is, $G \in \#(t)$ iff there exists a substitution $\sigma$ such that $\sigma(\overrightarrow{p q x y})=\overrightarrow{t s z w}$, $G=F \sigma$ and for all $j$ one has $\#\left(t_{i j}, A_{i j} \sigma\right), \neg \#\left(s_{i j}, B_{i j} \sigma\right), \#\left(z_{i j}, C_{i j}\right)$ and $\neg \#\left(w_{i j}, D_{i j} \sigma\right)$. All the sets \# $\left(t_{i j}\right)$ are finite by the induction hypothesis and all the sets $\#\left(z_{i j}\right)$ are finite due to the fact that $\mathcal{M}_{p}$ is finitely generated. Therefore, there are finitely many substitutions $\sigma$ satisfying the conditions above. Since $\operatorname{Var}(F) \in \operatorname{Var}\left(\delta_{i}^{+}\right) \cup \overrightarrow{p q x y}$, we conclude that $\#(t)$ is finite.

Finally, it remains to prove that $\mathcal{M}$ is reflexive as soon as $\mathcal{M}_{p}$ is. Suppose that $\#(t, G)$, we should prove that $\mathcal{M} \models G$. Induction on the construction of $t$. The cases when $t$ is a proof variable or $t \in T$ follow directly from the definition of $\#$ and the fact that $\mathcal{M}_{p}$ is reflexive. Let $t=f(\overrightarrow{t s}, \overrightarrow{z w})$ and $t \notin T$. Then by the definition of \# we have that \# $t, G)$ iff there exists a number $i$ and a substitution $\sigma$ such that $(\overrightarrow{p q x y}) \sigma=\overrightarrow{t s z w}, G=F \sigma$ and for all $j$ one has \# $\left(t_{i j}, A_{i j} \sigma\right), \neg \#\left(s_{i j}, B_{i j} \sigma\right), \#\left(z_{i j}, C_{i j}\right)$ and $\neg \#\left(w_{i j}, D_{i j} \sigma\right)$. By the induction hypothesis $\mathcal{M} \models A_{i j} \sigma$, therefore $\mathcal{M}=\llbracket t_{i j} \rrbracket A_{i j} \sigma$ and $\mathcal{M} \models \bigwedge \delta_{i} \sigma$. By the definition of an operation we have $\mathcal{L} \mathcal{P} \mathcal{S}_{0} \vdash \bigwedge \delta_{i} \rightarrow F$. Since $\mathcal{M}$ is an $\mathcal{L} \mathcal{P} \mathcal{S}_{0}$-model we conclude that $\mathcal{M} \models F \sigma$, that is, $\mathcal{M} \models G$.

\subsection{Arithmetical completeness of $\mathcal{L P} \mathcal{S}(\mathcal{F})$}

Theorem 3.14. (Arithmetical completeness.) Suppose that $\mathcal{F}$ consists of descriptive $q$-stable operations. For every formula $A$ if $\mathcal{L P} \mathcal{S}(\mathcal{F}) \forall A$ then there exists an interpretation $*$ of the language $\mathcal{L}(\mathcal{F})$ such that $A^{*}$ is false in the standard model.

Proof. The proof of soundness goes by standard induction on the derivation of $A$ in $\mathcal{L P S}(\mathcal{F})$.

To prove the completeness, suppose that $\mathcal{L P S}(\mathcal{F}) \not \forall A$. We have to show that there exists an interpretation $*$ such that $A^{*}$ is false. First we apply theorem 3.8 and find a finitely generated reflexive model $\mathcal{M}=(\#, v)$ such that $\mathcal{M} \forall A$. We are going to define an embedding of this model into Peano Arithmetic. We fix an injective Gödel numbering of the joint syntax of $\mathcal{L} \mathcal{P} \mathcal{S}(\mathcal{F})$ and Peano Arithmetic. We first consider an auxiliary evaluation $(\cdot)^{@}$ : for every sentence variable $S_{i}$, label variable $l$ and proof term $t$ put

$$
t^{@} \rightleftharpoons\ulcorner t\urcorner, \quad l^{@} \rightleftharpoons\ulcorner l\urcorner, \quad S_{i}^{@} \rightleftharpoons \begin{cases}i=i & \text { if } \mathcal{M} \models S_{i} \\ i=0 & \text { otherwise }\end{cases}
$$

We find the appropriate storage and proof predicates by the multiple fixed point equation. Since the model $\mathcal{M}$ is finitely generated, the relation " $\mathcal{M}=$ 
$\llbracket t \rrbracket F "$ is primitive recursive, thus, it can be represented by a $\Delta_{1}$ arithmetical formula. The function, which calculates $\left\ulcorner B^{@}\right\urcorner$ being given $\ulcorner\operatorname{Prf}\urcorner,\ulcorner\operatorname{Str}\urcorner$ and $\ulcorner B\urcorner(B$ an $\mathcal{L P \mathcal { S }}(\mathcal{F})$-formula), is primitive recursive too. Therefore, by the fixed point lemma for PA, there exist arithmetical $\Delta_{1}$ formulas $\operatorname{Prf}$, Str, such that the following formulas are provable in PA

$$
\begin{aligned}
& \operatorname{Prf}(x, y) \leftrightarrow \operatorname{Proof}(x, y) \vee \\
& \text { " } x=\ulcorner t\urcorner \text { and } y=\ulcorner B \text { @ }\urcorner \text { and } \mathcal{M} \models \llbracket t \rrbracket B " \\
& \operatorname{Str}(x, y) \leftrightarrow \operatorname{Store}(x, y) \vee \\
& \text { " } x=\ulcorner l\urcorner \text { and } y=\ulcorner B \text { @ }\urcorner \text { and } \mathcal{M} \models(l \ni B) \text { " }
\end{aligned}
$$

It is clear that the mapping $(\cdot)^{@}$ is injective, namely, for different formulas $B$ and $C$ we have $B^{\circledR} \neq C^{@}$.

Lemma 3.15. Let $\alpha$ be an arithmetical interpretation of the language $\mathcal{L} \mathcal{P} \mathcal{S}_{0}$ based on predicates $\operatorname{Prf}$ and $\operatorname{Str}$ defined by (FPE). Suppose that $A_{0}, \ldots, A_{n}$ are formulas in the language $\mathcal{L}_{0}$ and $\widetilde{A}_{0}, \ldots, \widetilde{A}_{n}$ are formulas in the language $\mathcal{L}(\mathcal{F})$. Suppose that

$$
A_{j}^{\alpha}=\widetilde{A}_{j}^{@} \text { for all } j .
$$

Then there exists a substitution $\sigma$ such that for every $\mathcal{L}_{0}$-formula $B$

$$
\text { if } \operatorname{Var}(B) \subseteq \bigcup_{j} \operatorname{Var}\left(A_{j}\right), \text { then } B^{\alpha}=(B \sigma)^{@} .
$$

Proof. For the sake of simplicity, we also allow in (5) equalities of the form $p^{\alpha}=t^{@}$ and $x^{\alpha}=y^{@}$ where $p$ is a proof variable, $t$ is a term and $x$, $y$ are label variables. We call formulas of $\mathcal{L}_{0}$ and proof and label variables expressions of the language $\mathcal{L}_{0}$; formulas of $\mathcal{L}(\mathcal{F})$, proof terms and label variables are expressions of $\mathcal{L}(\mathcal{F})$. So, the most general form of $(5)$ is $E_{j}^{\alpha}=$ $\widetilde{E_{j}}{ }^{@}(j=1, \ldots, n)$ where $E_{j}$ are expressions in the language $\mathcal{L}_{0}$ and $\widetilde{E_{j}}$ are expressions on the language $\mathcal{L}(\mathcal{F})$ of the same type.

We define the complexity of an expression $E$ in the language $\mathcal{L}_{0}$ as the total number of connectives in $E$ (namely, $|S|=0,|p|=|x|=0,|A \vee B|$ $=|A \wedge B|=|A \rightarrow B|=|A|+|B|+1,|\neg A|=|\llbracket p \rrbracket A|=|x \ni A|=|A|+1)$. We prove the existence of $\sigma$ satisfying (6) by induction on the parameter $\sum_{j}\left|E_{j}\right|$.

If $\sum_{j}\left|E_{j}\right|=0$ then all $E_{j}$ are variables. If some of these variables coincide, say $A_{l}=A_{k}$, we consequently obtain that $E_{l}^{\alpha}=E_{k}^{\alpha}$, whence $\widetilde{E}_{l}^{@}=\widetilde{E}_{k}^{@}$, and 
finally $\widetilde{E}_{l}=\widetilde{E}_{k}$ since the arithmetical evaluation @ is injective, namely, it maps different formulas of $\mathcal{L P S}(\mathcal{F})$ into different arithmetical formulas, and different proof and label variables into different numbers. Therefore, one can define a desired substitution $\sigma$ by $\sigma\left(E_{j}\right) \rightleftharpoons \tilde{E}_{j}$.

If $\sum_{j}\left|E_{j}\right|>0$ then there exists a formula, say $A_{j}$, which is not a sentence variable. In this case the main connective of $A_{j}$ coincides with the main connective of $A_{j}^{\alpha}$, which in turn coincides with the main connective of $\widetilde{A}_{j}^{@}$. Note that the interpretation @ maps sentence variables to atomic arithmetical formulas, therefore, the main connective of $\widetilde{A}_{j}^{@}$ coincides with the main connective of $\widetilde{A}_{j}$. Therefore, we can replace the condition $\widetilde{A}_{j}^{@}=A_{j}^{\alpha}$ in $(5)$ by the corresponding equalities for the components of $A_{j}$. Doing so, we decrease the induction parameter. For example, consider the most interesting case is $A_{j}=\llbracket p \rrbracket B_{j}, \widetilde{A}_{j}=\llbracket t \rrbracket \widetilde{B}_{j}$. In this case the corresponding equation in (5) has the form $\operatorname{Prf}\left(p^{\alpha},\left\ulcorner B_{j}^{\alpha}\right\urcorner\right)=\operatorname{Prf}\left(\ulcorner t\urcorner,\left\ulcorner\widetilde{B}_{j}^{@}\right\urcorner\right)$. We can replace this equality by two new ones: $p^{\alpha}=t^{@}$ and $B_{j}^{\alpha}=\widetilde{B}_{j}^{@}$.

Lemma 3.16. For every formula $C$

$$
\begin{aligned}
& \mathcal{M} \models C \Rightarrow \mathbf{P A} \vdash C^{@} ; \\
& \mathcal{M} \forall C \Rightarrow \mathbf{P A} \vdash \neg C^{@} .
\end{aligned}
$$

Proof. Induction on the formula $C$. The induction base when $C$ is a propositional variable is a direct corollary of the definition of @. The case of boolean connectives immediately follows from the induction hypothesis.

Suppose that $C$ has the form $\llbracket t \rrbracket B$. If $\mathcal{M}=C$ then by the definition of $\operatorname{Prf}$ we conclude that $\operatorname{Prf}\left(\ulcorner t\urcorner,\left\ulcorner B^{@}\right\urcorner\right)$ is true, whence PA $\vdash \operatorname{Prf}\left(\ulcorner t\urcorner,\left\ulcorner B^{@}\right\urcorner\right)$ that is PA $\vdash C^{@}$. If $\mathcal{M} \forall \forall C$ then $\operatorname{Prf}\left(\ulcorner t\urcorner,\left\ulcorner B^{@}\right\urcorner\right)$ is false because of the injectivity of the evaluation $(\cdot)^{@}$. Therefore PA $\vdash \neg \operatorname{Prf}\left(\ulcorner t\urcorner,\left\ulcorner B^{@}\right\urcorner\right)$, that is, $\mathbf{P A} \vdash \neg C^{@}$. The remaining case when $C$ has the form $x \ni B$ can be treated similarly.

Lemma 3.17. Prf is a normal proof predicate, Str is a storage predicate.

Proof. From properties of finitely generated models and definition of $\operatorname{Prf}$, Str it follows that for every proof term $t$ and label variable $l$ the sets $T h(\ulcorner t\urcorner)$ and $\operatorname{Cont}(\ulcorner l\urcorner)$ are finite and the functions $x \mapsto T h(x)$ and $x \mapsto \operatorname{Cont}(x)$ are recursive for Prf nd Str. 
It only remains to verify that if $\operatorname{Prf}(n,\ulcorner\varphi\urcorner)$ is true then $\mathbf{P A} \vdash \varphi$. Suppose that $\operatorname{Prf}(n,\ulcorner\varphi\urcorner)$. By the definition of $\operatorname{Prf}$ there are two possibilities. The first case when Proof $(n,\ulcorner\varphi\urcorner)$ is trivial. For the second case suppose that $n=\ulcorner t\urcorner, \varphi=B^{@}$ and $\mathcal{M}=\llbracket t \rrbracket B$. In this case $\mathcal{M} \models B$ whence PA $\vdash B^{@}$ by lemma 3.16.

Now we can define interpretation of operations on proofs. Consider an operation $f$ described by the specification axiom $\bigvee_{i} \delta_{i} \rightarrow \llbracket f(\overrightarrow{p, q}, \overrightarrow{x, y}) \rrbracket F$. Let $f^{@}$ be a total recursive function which satisfies this specification axiom for the standard predicates Proof, Store. The following function which converts Prf-proofs and Str-labels into proofs and labels according to Proof and Store is total recursive:

$$
c v(x) \rightleftharpoons \begin{cases}\mu y . \forall z(\operatorname{Prf}(x, z) \leftrightarrow \operatorname{Proof}(y, z)) & \text { if } x=\ulcorner t\urcorner \text { for } t \in \operatorname{Tm}(\mathcal{F}) \\ \mu y . \forall z(\operatorname{Str}(x, z) \leftrightarrow \operatorname{Store}(y, z)) & \text { if } x=\ulcorner l\urcorner \text { for } l \in \operatorname{LVar} \\ x & \text { otherwise }\end{cases}
$$

For every functional symbol $f \in \mathcal{F}$ let $f^{@}$ be a total recursive function satisfying $\operatorname{Spec}(f)$ for Proof and Str. We define total recursive function $f^{*}$ as follows:

$f^{*}(\vec{n}, \vec{m}) \rightleftharpoons \begin{cases}\ulcorner f(\vec{n}, \vec{m})\urcorner & \text { if for all } i, j n_{i j}=\left\ulcorner t_{i j}\right\urcorner, m_{i j}=\left\ulcorner x_{i j}\right\urcorner, \\ f^{@}\left(\overrightarrow{c v\left(n_{i j}\right)}, \overrightarrow{c v\left(m_{i j}\right)}\right) & \text { where } t_{i j} \in \operatorname{Tm}(\mathcal{F}), x_{i j} \in \operatorname{LVar} \\ \text { otherwise }\end{cases}$

Lemma 3.18. Functions $f^{*}$ defined above satisfy the corresponding specification axioms $\operatorname{Spec}(f)$.

Proof. Consider a specification axiom $\bigvee_{i}\left(\wedge \delta_{i}\right) \rightarrow \llbracket f(\vec{p}, \vec{x}) \rrbracket F$. Let $\alpha=$ $\left(\operatorname{Prf}, \operatorname{Str},(\cdot)^{\alpha}\right)$ be an arithmetical interpretation. Suppose that $\delta_{i}^{\alpha}$ is true for some $i$. Then there are two possibilities: 1) for every $i, j$ it is true that $p_{i j}^{\alpha}=\left\ulcorner t_{i j}\right\urcorner, q_{i j}^{\alpha}=\left\ulcorner s_{i j}\right\urcorner, x_{i j}^{\alpha}=\left\ulcorner z_{i j}\right\urcorner, y_{i j}^{\alpha}=\left\ulcorner w_{i j}\right\urcorner$ for some terms $t_{i j}, s_{i j}$ and label variables $z_{i j}, w_{i j}$ or 2 ) it is not true for some $i, j$. The second case is trivial.

Let us consider the first possibility. In this case there exists $i$ such that for all $j$ the formulas $\operatorname{Prf}\left(\left\ulcorner t_{i j}\right\urcorner,\left\ulcorner A_{i j}^{\alpha}\right\urcorner\right)$ and $\operatorname{Str}\left(\left\ulcorner z_{i j}\right\urcorner,\left\ulcorner C_{i j}^{\alpha}\right\urcorner\right)$ are true and $\operatorname{Prf}\left(\left\ulcorner s_{i j}\right\urcorner,\left\ulcorner B_{i j}^{\alpha}\right\urcorner\right)$ and $\operatorname{Str}\left(\left\ulcorner w_{i j}\right\urcorner,\left\ulcorner D_{i j}^{\alpha}\right\urcorner\right)$ are false. We fix $i$ as above. By (FPE) we can conclude that for all $j$ there exist formulas $\widetilde{A}_{i j}, \widetilde{C}_{i j}$ such that

$$
\begin{array}{cl}
\mathcal{M} \models \llbracket t_{i j} \rrbracket \widetilde{A}_{i j}, & \mathcal{M}=x_{i j} \ni \widetilde{C}_{i j} \quad \text { and } \\
A_{i j}^{\alpha}=\widetilde{A}_{i j}^{@}, & C_{i j}^{\alpha}=\widetilde{C}_{i j}^{@} .
\end{array}
$$


According to lemma 3.15 there exists a substitution $\sigma$ such that for every formula $B$ satisfying the condition $\operatorname{Var}(B) \subseteq \bigcup_{i} \operatorname{Var}\left(\delta_{i}^{+}\right)$one has $B^{\alpha}=$ $(B \sigma)^{@}$. In particularly, in view of $(9)$ we have $\widetilde{A}_{i j}^{@}=A_{i j}^{\alpha}=\left(A_{i j} \sigma\right)^{@}$, and the same for $C_{i j}$. Since interpretation @ is injective, this yields for all $j$

$$
A_{i j} \sigma=\widetilde{A}_{i j}, C_{i j} \sigma=\widetilde{C}_{i j}
$$

Combining (10) and (8) we obtain that $\mathcal{M} \models \llbracket t_{i j} \rrbracket\left(A_{i j} \sigma\right), \mathcal{M} \models\left(x_{i j} \ni\left(C_{i j} \sigma\right)\right)$.

Let us show that for all $j$ we have $\mathcal{M} \forall \llbracket \llbracket s_{i j} \rrbracket\left(B_{i j} \sigma\right)$ and $\mathcal{M} \not \models\left(y_{i j} \ni D_{i j} \sigma\right)$. Suppose for the converse that for some $j$ one has $\mathcal{M} \models \llbracket s_{i j} \rrbracket\left(B_{i j} \sigma\right)$. By the definition of a $q$-stable descriptive operation we have $\operatorname{Var}(B) \subseteq \operatorname{Var}\left(\delta_{i}^{+}\right)$ whence by lemma 3.15 we obtain $B_{i j}^{\alpha}=\left(B_{i j} \sigma\right)^{@}$ From $\mathcal{M} \models \llbracket s_{i j} \rrbracket\left(B_{i j} \sigma\right)$ by $(\mathrm{FPE})$ it follows that $\operatorname{Prf}\left(\left\ulcorner s_{i j}\right\urcorner,\left\ulcorner\left(B_{i j} \sigma\right)^{@}\right\urcorner\right)$, that is, $\operatorname{Prf}\left(\left\ulcorner s_{i j}\right\urcorner,\left\ulcorner B_{i j}^{\alpha}\right\urcorner\right)$, contradiction. The case of the storage operator can be treated similarly.

Now we have $\mathcal{M} \models \bigwedge\left(\delta_{i} \sigma\right)$. By the correctness of $\mathcal{L} \mathcal{P} \mathcal{S}(\mathcal{F})$ with respect to the symbolic models we conclude that $\mathcal{M} \models \llbracket f(\vec{t}, \vec{x}) \rrbracket(F \sigma)$ whence $\operatorname{Prf}\left(\ulcorner f(\vec{t}, \vec{x})\urcorner,\left\ulcorner(F \sigma)^{@}\right\urcorner\right)$ by the definition of $\operatorname{Prf}$. Since $\operatorname{Var}(F) \subseteq \operatorname{Var}\left(\delta_{i}^{+}\right) \cup$ $\overrightarrow{p q x y}$, we have that $(F \sigma)^{@}=F^{\alpha}$. From the definition of $f^{*}$ we derive $f^{*}\left(\overrightarrow{t^{\alpha}}, \vec{x}^{\alpha}\right)=\ulcorner f(\vec{t}, \vec{x})\urcorner$ whence $\operatorname{Prf}\left(f^{*}\left(\vec{t}^{\alpha}, \vec{x}^{\alpha}\right),\left\ulcorner F^{\alpha}\right\urcorner\right)$.

Now we define the interpretation $*=\left(\operatorname{Prf}, \operatorname{Str}, \mathcal{F}^{*},(\cdot)^{*}\right)$ where for every $V \in \operatorname{Var}$

$$
V^{*} \rightleftharpoons V^{@} .
$$

By induction on the term $t$ we can show that $t^{*}=t^{@}$. Therefore, for every formula $F$ we have $F^{*}=F^{@}$. So from lemma 3.16 we deduce that for every formula $C$

$$
\begin{aligned}
& \mathcal{M} \models C \Rightarrow \text { PA } \vdash C^{*} \\
& \mathcal{M} \forall C \Rightarrow \text { PA } \vdash \neg C^{*} .
\end{aligned}
$$

Since $\mathcal{M} \not A A$, we conclude that $\mathbf{P A} \vdash \neg A^{*}$.

\section{Acknowledgement}

I am thankful to Sergei Artemov who introduced me to the subject and provided advise and encouragement. I am also grateful to Vladimir Krupski for numerous discussions on the subject and his helpful remarks. 


\section{References}

[1] S. Artemov, Uniform provability realization of intuitionistic logic, modality and $\lambda$-terms, Electronic Notes in Theoretical Computer Science 23 (1999).

[2] S. Artemov, Explicit provability and constructive semantics, Bulletine of Symbolic Logic 7 (2001), 1-36.

[3] S. Artemov, V. Krupski, Data storage interpretation of labeled modal logic, Annals of Pure and Applied Logic 78 (1996), 57-71.

[4] V. N. Krupski, Operational logic of proofs with functionality condition on proof predicate, Lecture notes in computer science 1234 (1997), 167-177.

[5] V. Krupski, The single-conclusion proof logic and inference rules specification, Annals of Pure and Applied Logic 113 (2002), 181-206.

[6] V. Krupski, Referential logic of proofs, Theoretical Computer Science, to appear.

[7] R. Kuznets, On the complexity of explicit modal logics, Computer Science Logic 2000, Lecture Notes in Computer Science 1862 (2000), 371-383.

[8] A. Mkrtychev, Models for the Logic of Proofs, Lecture Notes in Computer Science, v. 1234 (1997), Logical Foundations of Computer Science '97, Yaroslavl', pp. 266-275.

[9] N.Rubtcova, T.Yavorskaya, Operations on proofs and labels, Journal of applied nonclassical logic, to appear.

[10] C. Smorynsky, Self-reference and Modal Logic, Springer, New York, 1985.

[11] T. Yavorskaya (Sidon), Logic of proofs and provability, Annals of Pure and Applided logic 113 (2002), 345-372. 